TRANSACTIONS OF THE

AMERICAN MATHEMATICAL SOCIETY

Volume 360, Number 11, November 2008, Pages 6149-6178

S 0002-9947(08)04509-1

Article electronically published on May 29, 2008

\title{
STABILITY CONDITIONS AND CREPANT SMALL RESOLUTIONS
}

\author{
YUKINOBU TODA
}

\begin{abstract}
In this paper, we describe the spaces of stability conditions on the triangulated categories associated to three dimensional crepant small resolutions. The resulting spaces have chamber structures such that each chamber corresponds to a birational model together with a special Fourier-Mukai transform. We observe that these spaces are covering spaces over certain open subsets of finite dimensional vector spaces and determine their deck transformations.
\end{abstract}

\section{INTRODUCTION}

For a triangulated category $\mathcal{D}$, the notion of stability conditions on $\mathcal{D}$ was introduced by T. Bridgeland [5] to give a mathematical framework for Douglas's notion of $\Pi$-stability [13], 14. Roughly speaking, Bridgeland's stability condition $(Z, \mathcal{P})$ on $\mathcal{D}$ consists of a group homomorphism $Z$ and full subcategories,

$$
Z: K(\mathcal{D}) \longrightarrow \mathbb{C}, \quad \mathcal{P}(\phi) \subset \mathcal{D} \quad(\phi \in \mathbb{R}),
$$

where $K(\mathcal{D})$ is the Grothendieck group of $\mathcal{D}$, and this pair satisfies some axioms. Given data as above, the objects of the subcategory $\mathcal{P}(\phi)$ are called semistable of phase $\phi$, and this gives a generalization of the classical notion of semistable sheaves with a fixed slope on a smooth projective curve. In [5], T .Bridgeland showed that the set of locally finite stability conditions $\operatorname{Stab}(\mathcal{D})$ has a natural topology and proposed to study this space as a new categorical invariant. After he wrote the paper [5], some examples were computed in [7], 8], [10], 29], 27], [26], [18, [2]. The purpose of this paper is to describe the spaces of stability conditions on some triangulated categories associated to three dimensional crepant small resolutions.

1.1. Crepant small resolutions. Let $Y=\operatorname{Spec} R$ for a noetherian local complete Gorenstein $\mathbb{C}$-algebra $R$ of dimension three, and $0 \in Y$ be the closed point. Assume that $Y$ admits a resolution of singularities

$$
f: X \rightarrow Y=\operatorname{Spec} R
$$

which is an isomorphism in codimension one. Then $f: X \rightarrow Y$ is called a crepant small resolution. It is well known that $\omega_{X}=f^{*} \omega_{Y}$ and the exceptional locus $C=$ $\bigcup_{i=1}^{N} C_{i}$ is a tree of rational curves. Let $D^{b}(X)$ be the bounded derived category

Received by the editors October 11, 2006 and, in revised form, February 26, 2007 and March 12, 2007.

2000 Mathematics Subject Classification. Primary 14J32, 14E30, 18E30.

(C)2008 American Mathematical Society 
of coherent sheaves on $X$. We define the triangulated subcategory $\mathcal{D}_{X / Y} \subset D^{b}(X)$ as follows:

$$
\mathcal{D}_{X / Y}:=\left\{E \in D^{b}(X) \mid \operatorname{Supp}(E) \subset C\right\} .
$$

The purpose of this paper is to study the space of stability conditions on $\mathcal{D}_{X / Y}$. Define the space $\operatorname{Stab}(X / Y)$ by

$$
\operatorname{Stab}(X / Y):=\operatorname{Stab}\left(\mathcal{D}_{X / Y}\right) .
$$

The simplest example is given by the morphism,

$$
f: X \longrightarrow Y=\operatorname{Spec} \mathbb{C}[[x, y, z, w]] /(x y-z w),
$$

which is the blowing up by the ideal $(x, z) \subset \mathcal{O}_{Y}$. In this case, the exceptional locus is a single rational curve, whose normal bundle is $\mathcal{O}_{C}(-1) \oplus \mathcal{O}_{C}(-1)$. Taking the blow-up by the ideal $(x, w) \subset \mathcal{O}_{Y}$ gives another resolution $g: W \rightarrow Y$, and the diagram

$$
X \stackrel{f}{\longrightarrow} Y \stackrel{g}{\longleftarrow} W
$$

is an example of a flop [25, Definition 6.10]. A new feature in studying $\operatorname{Stab}(X / Y)$ is that one has to take account of all the crepant small resolutions of $Y$, such as flops. We would like to explain this in two different contexts: string theory and minimal model theory.

1.2. Viewpoint from string theory. Here we give a rough picture of the space $\operatorname{Stab}(X / Y)$, from the viewpoint of string theory. We use notions of string theory, for example stringy Kähler moduli spaces, and SCFT. These notions are explained in 4 . Section 2]. For instance, assume $X$ is a projective Calabi-Yau 3-fold, $\mathcal{D}=D^{b}(X)$, and $\mathcal{M}_{K}(X)$ is the stringy Kähler moduli space [4, (2.2)]. The space $\mathcal{M}_{K}(X)$ is a subspace of the moduli space of SCFT, and the associated topological B-twists are unchanged along $\mathcal{M}_{K}(X)$. Moreover if $\hat{X}$ is a mirror of $X, \mathcal{M}_{K}(X)$ is supposed to be isomorphic to the moduli space of complex structures on $\hat{X}$. The space of stability conditions was introduced in order to understand $\mathcal{M}_{K}(X)$ mathematically. More precisely, it is believed that the quotient space of $\operatorname{Stab}(\mathcal{D})$ by the actions of $\operatorname{Aut}(\mathcal{D})$ and $\mathbb{C}$ contains $\mathcal{M}_{K}(X)$ [4 Remark 3.9].

The moduli space of complexified Kähler forms $\beta+i \omega \in H^{2}(X, \mathbb{C})$ forms an open subset $\mathcal{U}_{X} \subset \mathcal{M}_{K}(X)$, which physicists call the neighborhood of the large volume limit. The important point is that there might be another topologically distinct Calabi-Yau 3-fold $W$ such that $\mathcal{U}_{W}$ is contained in $\mathcal{M}_{K}(X)$ in the moduli space of SCFT. In this case, the associated B-twists from the data of $X$ and $W$ are equivalent; therefore their categories of D-branes are also equivalent. Mathematically this means that there exists an equivalence of the bounded derived categories of coherent sheaves,

$$
\Phi: D^{b}(W) \longrightarrow D^{b}(X) .
$$

In the language of algebraic geometry, $W$ is called a Fourier-Mukai partner of $X$ and a flop gives one example ([3], 6], 11, 20]). Therefore in describing $\mathcal{M}_{K}(X)$, we have to take into account the neighborhoods of the large volume limits corresponding to several Fourier-Mukai partners.

In fact in the case of a flop $X \rightarrow Y \leftarrow W$, P. Aspinwall [1, Figure 2] describes the localized picture of $\mathcal{M}_{K}(X)$ assuming all the curves in $X$ except the flopping curve $C \subset X$ are quite "big". The resulting picture is a 2 -sphere minus three points, $\mathcal{U}_{X}, \mathcal{U}_{W}$ are disjoint and their union is dense in the sphere. The string theory has singularities at one of the deleted points, and the other two points are large 
volume limits corresponding to $X$ and $W$. Thus one should have a similar picture in the context of Bridgeland's stability conditions. It seems that our localized category $\mathcal{D}_{X / Y}$ and the space $\operatorname{Stab}(X / Y)$ provide the right framework; thus we expect $\operatorname{Stab}(X / Y)$ to be described via Fourier-Mukai partners such as flops.

1.3. Viewpoint from minimal model theory. Our interest also comes from birational geometry, especially the minimal model program, written simply MMP ([25], 22]). The MMP is a program aimed at finding a good birational model for a given projective variety, by contracting extraneous rational curves. The output is either a minimal model or Mori fiber space.

One of the points where the three dimensional MMP differs from the two dimensional one is that birational minimal models are not necessarily unique, but connected by a sequence of flops [25, Theorem 6.38]. The philosophy of Y. Kawamata [19] is that one can capture the set of birational minimal models via a chamber structure on the movable cone [19, Definition 1.1]. According to [19, Theorem 2.3], chambers on the movable cone are given by the ample cones of birational minimal models.

Now let us consider two birational three dimensional minimal models $W \rightarrow X$. Then there exists an equivalence of bounded derived categories of coherent sheaves ([3], 6], [1], 20]),

$$
\Phi: D^{b}(W) \longrightarrow D^{b}(X) .
$$

Let $\operatorname{Stab}(X)$ be the space of stability conditions on $D^{b}(X)$. As a substitute for the ample cone, one expects to find a certain region $U_{X} \subset \operatorname{Stab}(X)$, which corresponds to the neighborhood of the large volume limit in string theory. Then one can transfer stability conditions in $U_{W} \subset \operatorname{Stab}(W)$ by the equivalence $\Phi$ to get the region $U(W, \Phi) \subset \operatorname{Stab}(X)$. In summary, for a birational minimal model $W$ and an equivalence $\Phi$ as above, one obtains the correspondence

$$
(W, \Phi) \longmapsto U(W, \Phi) \subset \operatorname{Stab}(X) .
$$

This picture is quite similar to the picture of the movable cone [19, Theorem 2.3]. Thus we guess the existence of the chamber structure on $\operatorname{Stab}(X)$, which enables us to capture the pair $(W, \Phi)$ as above. Unfortunately, there are some technical issues in working with Bridgeland's stability conditions for the derived categories of projective 3 -folds. In particular we do not know how to construct examples of stability conditions in this case. (Also see the last part of [4, Section 4].) Despite this problem, our category $\mathcal{D}_{X / Y}$ is quite amenable to studying stability conditions, and also sufficient for realizing our purpose.

1.4. The main results. Let $f: X \rightarrow Y$ be a crepant small resolution as in the first part of this introduction. For some technical reasons, we put the following additional assumption:

- There is a hyperplane section $0 \in Y_{0} \subset Y$ such that its pull-back $X_{0}:=$ $f^{-1}\left(Y_{0}\right)$ is smooth.

First we give the standard region in Definition 4.2 below,

$$
U_{X} \subset \operatorname{Stab}(X / Y),
$$

which should correspond to the neighborhood of the large volume limit. Unfortunately, $U_{X}$ is not an open subset of $\operatorname{Stab}(X / Y)$, but open in the subspace of 
$\operatorname{Stab}(X / Y)$ which we call the normalized stability conditions,

$$
\operatorname{Stab}_{\mathrm{n}}(X / Y):=\left\{\sigma=(Z, \mathcal{P}) \in \operatorname{Stab}(X / Y) \mid Z\left(\left[\mathcal{O}_{x}\right]\right)=-1\right\} .
$$

In this paper, we give a description of the normalized version of the stability conditions. Because other stability conditions are obtained by the actions of the additive group $\mathbb{C}$ from the normalized stability conditions, it is enough to study $\operatorname{Stab}_{\mathrm{n}}(X / Y)$ for our purpose. Here the action of $\mathbb{C}$ is as follows: for $\lambda \in \mathbb{C}$ and $\sigma=(Z, \mathcal{P}) \in \operatorname{Stab}(X / Y), \lambda(\sigma)=\left(Z^{\prime}, \mathcal{P}^{\prime}\right)$ with $\mathcal{P}^{\prime}(\phi)=\mathcal{P}(\phi+\operatorname{Re} \lambda)$ and $Z^{\prime}(E)=$ $\exp (-i \pi \lambda) Z(E)$. Let $\operatorname{Stab}_{\mathrm{n}}^{\circ}(X / Y)$ be the connected component of $\operatorname{Stab}_{\mathrm{n}}(X / Y)$ which contains $U_{X}$.

Next we introduce the set $\operatorname{FM}(X)$ to be the set of pairs $(W, \Phi)$, where $g: W \rightarrow Y$ is a crepant small resolution, and

$$
\Phi: D^{b}(W) \longrightarrow D^{b}(X)
$$

gives an equivalence of derived categories, such that $\Phi$ is given in a special way, as described in Definition 3.13 below. By transferring the open set $U_{W} \subset \operatorname{Stab}_{\mathrm{n}}(W / Y)$ through the equivalence $\Phi$, we give the following open set in Definition 4.7

$$
U(W, \Phi) \subset \operatorname{Stab}_{\mathrm{n}}(X / Y) .
$$

The following theorem realizes our purpose, that is, the existence of the desired chamber structure on the space of stability conditions.

Theorem 1.1. We have the following union of chambers:

$$
\mathcal{M}:=\bigcup_{(W, \Phi) \in \mathrm{FM}(\mathrm{X})} U(W, \Phi) \subset \operatorname{Stab}_{\mathrm{n}}^{\circ}(X / Y),
$$

such that two chambers are either disjoint or equal. Moreover we have $\overline{\mathcal{M}}=$ $\operatorname{Stab}_{\mathrm{n}}^{\circ}(X / Y)$.

By using the chamber structure given in Theorem 1.1, we show $\operatorname{Stab}_{\mathrm{n}}^{\circ}(X / Y)$ is a regular covering space over a certain open subset of a finite dimensional complex vector space. Let $\Lambda_{f}$ be the root lattice associated to the exceptional locus of $f$. We denote by $N^{1}(X / Y)$ the group of numerical classes of $\mathbb{R}$-divisors and $N^{1}(X / Y)_{\mathbb{C}}:=$ $N^{1}(X / Y) \otimes_{\mathbb{R}} \mathbb{C}$. Then the elements of $N^{1}(X / Y)_{\mathbb{C}}$ are regarded as complex functions on $\Lambda_{f}$. Let $V\left(\Lambda_{f}\right)$ be the set of roots of $\Lambda_{f}$. For a root $v \in V\left(\Lambda_{f}\right)$ and $k \in \mathbb{Z}$, define $H_{v}$ as follows:

$$
H_{v}:=\left\{\beta+i \omega \in N^{1}(X / Y)_{\mathbb{C}} \mid(\beta+i \omega) v \in \mathbb{Z}\right\} .
$$

Theorem 1.2. The map

$$
\mathcal{Z}_{X}: \operatorname{Stab}_{\mathrm{n}}^{\circ}(X / Y) \longrightarrow N^{1}(X / Y)_{\mathbb{C}} \backslash \bigcup_{v \in V\left(\Lambda_{f}\right)} H_{v}
$$

defined by sending a stability condition to its central charge, is a regular covering map.

The results of this paper together with some developments on derived categories and stability conditions are reviewed in T. Bridgeland's manuscript [9] for the ICM talk in 2006. 


\section{Generalities}

In this paper, all the schemes are defined over $\mathbb{C}$. For a scheme $X$, we denote by $\operatorname{Coh}(X)$ and $D^{b}(X)$ the Abelian category of coherent sheaves and its bounded derived category, respectively. The shift functor on $D^{b}(X)$ is denoted by [1]. Also for a subscheme $Z \subset X$, we denote by $\operatorname{Coh}_{Z}(X) \subset \operatorname{Coh}(X)$ the subcategory whose objects are supported on $Z$. For an object $E \in D^{b}(X)$, its support is defined by

$$
\operatorname{Supp}(E):=\bigcup_{p \in \mathbb{Z}} \operatorname{Supp} H^{p}(E) \subset X .
$$

For a triangulated category $\mathcal{D}$, its $K$-group is denoted by $K(\mathcal{D})$.

2.1. Stability conditions on triangulated categories. The notion of stability conditions on triangulated categories is introduced in [5] to give the mathematical framework for Douglas's work on П-stability (13], [14]). Here we collect the basic definitions and results in [5].

Definition 2.1. A stability condition on a triangulated category $\mathcal{D}$ consists of data $\sigma=(Z, \mathcal{P})$, where $Z: K(\mathcal{D}) \rightarrow \mathbb{C}$ is a linear map, and $\mathcal{P}(\phi) \subset \mathcal{D}$ is a full additive subcategory for each $\phi \in \mathbb{R}$, which satisfy the following:

- $\mathcal{P}(\phi+1)=\mathcal{P}(\phi)[1]$.

- If $\phi_{1}>\phi_{2}$ and $A_{i} \in \mathcal{P}\left(\phi_{i}\right)$, then $\operatorname{Hom}\left(A_{1}, A_{2}\right)=0$.

- If $E \in \mathcal{P}(\phi)$ is non-zero, then $Z(E)=m(E) \exp (i \pi \phi)$ for some $m(E) \in \mathbb{R}_{>0}$.

- For a non-zero object $E \in \mathcal{D}$, we have the following collection of triangles:
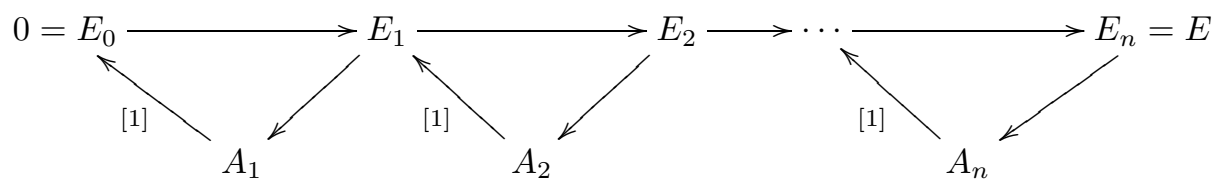

such that $A_{j} \in \mathcal{P}\left(\phi_{j}\right)$ with $\phi_{1}>\phi_{2}>\cdots>\phi_{n}$.

Here $Z$ is called the central charge. Each $\mathcal{P}(\phi)$ is an Abelian category, the nonzero objects of $\mathcal{P}(\phi)$ are called semistable of phase $\phi$, and simple objects of $\mathcal{P}(\phi)$ are called stable. The objects $A_{j}$ are called semistable factors of $E$ with respect to $\sigma$. The following proposition is useful in constructing stability conditions.

Proposition 2.2 ([5, Proposition 4.2]). Giving a stability condition on $\mathcal{D}$ is equivalent to giving a heart of a bounded t-structure $\mathcal{A} \subset \mathcal{D}$, and a group homomorphism $Z: K(\mathcal{D}) \rightarrow \mathbb{C}$, called the stability function, such that for a non-zero object $E \in \mathcal{A}$ one has

$$
Z(E) \in\{r \exp (i \pi \phi) \mid r>0,0<\phi \leq 1\}
$$

and the pair $(Z, \mathcal{A})$ satisfies the Harder-Narasimhan property.

For the Harder-Narasimhan property, we refer to [5, Definition 2.3]. Also, a necessary condition for this property is found in [5, Proposition 2.4]. Given data $(Z, \mathcal{A})$ as above and a non-zero object $E \in \mathcal{A}$, we define $\phi(E) \in(0,1]$ such that $Z(E) \in \mathbb{R}_{>0} \exp (i \pi \phi(E))$ uniquely. We call $\phi(E)$ the phase of $E$.

Remark 2.3. The condition (b) of [5, Proposition 2.4] is satisfied if $\mathcal{A}$ is noetherian; i.e. for $E \in \mathcal{A}$, there are no infinite sequences of subobjects in $\mathcal{A}$,

$$
E_{1} \subset E_{2} \subset \cdots \subset E_{j} \subset \cdots \subset E .
$$


Therefore in order to check the Harder-Narasimhan property, it is enough to check that $\mathcal{A}$ is noetherian and the condition (a) of [5, Proposition 2.4]; i.e. there is no infinite sequence of subobjects in $\mathcal{A}$,

$$
\cdots \subset E_{j+1} \subset E_{j} \subset \cdots \subset E_{2} \subset E_{1},
$$

with $\phi\left(E_{j+1}\right)>\phi\left(E_{j}\right)$ for all $j$.

The set of stability conditions which satisfies the technical condition local finiteness [5, Definition 5.7] is denoted by $\operatorname{Stab}(\mathcal{D})$. It is shown in [5, Section 6] that $\operatorname{Stab}(\mathcal{D})$ has a natural topology. Forgetting the information of $\mathcal{P}$, we have the map

$$
\mathcal{Z}: \operatorname{Stab}(\mathcal{D}) \longrightarrow \operatorname{Hom}_{\mathbb{Z}}(K(\mathcal{D}), \mathbb{C}) .
$$

Theorem 2.4 ([5, Theorem 1.2]). For each connected component $\Sigma \subset \operatorname{Stab}(\mathcal{D})$, there exists a linear subspace $V(\Sigma) \subset \operatorname{Hom}_{\mathbb{Z}}(K(\mathcal{D}), \mathbb{C})$, such that $\mathcal{Z}$ restricts to a local homeomorphism, $\mathcal{Z}: \Sigma \rightarrow V(\Sigma)$.

In general $\operatorname{Stab}(\mathcal{D})$ is infinite dimensional, so we usually consider only numerical stability conditions as in [5], 7]. But if we assume $K(\mathcal{D})$ is finitely generated, Theorem 2.4 implies each connected component $\Sigma \subset \operatorname{Stab}(\mathcal{D})$ is a complex manifold.

\section{Geometry on Crepant small Resolutions}

Let $f: X \rightarrow Y=\operatorname{Spec} R$ be a three dimensional crepant small resolution as in the introduction. The exceptional locus $C \subset X$ is a tree of rational curves,

$$
C=C_{1} \cup C_{2} \cup \cdots \cup C_{N},
$$

with each $C_{i}$ isomorphic to $\mathbb{P}^{1}$. (See for example [12, Lemma 3.4.1].) In this paper, we put the following additional assumption.

- There exists a hyperplane section $0 \in Y_{0} \subset Y$ such that its pull-back $X_{0}:=f^{-1}\left(Y_{0}\right)$ is smooth.

In general, it is known that a general hyperplane section $Y_{0}$ is a rational double point, and $X_{0} \rightarrow Y_{0}$ is a partial resolution; i.e. the minimal resolution $X_{0}^{\prime} \rightarrow Y_{0}$ factors through $X_{0}^{\prime} \rightarrow X_{0} \rightarrow Y_{0}$. (See [28, (1.1), (1.14)].) Also it is known that for a given crepant small resolution $f: X \rightarrow Y$, there exists a finite map $Y^{\prime} \rightarrow Y$ such that $Y^{\prime}$ admits a crepant small resolution which satisfies the above assumption. (See [25, Theorem 4.28].) The above assumption will be required in Lemma 3.8 . Subsection 5.1 and Lemma 6.3. Of course this assumption is satisfied in the case of the resolution of the ordinary double point described in the introduction. As in the introduction, we define $\mathcal{D}_{X / Y}$ to be

$$
\mathcal{D}_{X / Y}:=\left\{E \in D^{b}(X) \mid \operatorname{Supp} E \subset C\right\} .
$$

We collect some notation and known results on this resolution and give some lemmas. All the lemmas in this section will be proved in Section 6 .

3.1. Terminology and results from birational geometry. Here we introduce standard terminology in birational geometry, for example used in 19, Definition 1.1]. Two divisors $D_{1}, D_{2}$ on $X$ are called numerically equivalent over $Y$ if and only if $D_{1} \cdot C_{i}=D_{2} \cdot C_{i}$ for all $1 \leq i \leq N$. Similarly, two 1-cycles $Z_{1}, Z_{2}$ on $X$ contracted by $f$ are numerically equivalent if and only if $D \cdot Z_{1}=D \cdot Z_{2}$ for every divisor $D$ on $X$. 
Definition 3.1. We define the $\mathbb{R}$-vector spaces $N^{1}(X / Y), N_{1}(X / Y)$ to be $N^{1}(X / Y):=\{$ Divisors on $X\} /($ numerical equivalence over $Y) \otimes_{\mathbb{Z}} \mathbb{R}$, $N_{1}(X / Y):=\{$ One cycles on $X$ contracted by $f\} /($ numerical equivalence $) \otimes_{\mathbb{Z}} \mathbb{R}$.

By the definition, one has the perfect pairing,

$$
N^{1}(X / Y) \times N_{1}(X / Y) \ni(D, Z) \longmapsto D \cdot Z \in \mathbb{R} .
$$

Moreover since we are assuming $Y$ is complete, there exist divisors $D_{i}$ on $X$ for $1 \leq i \leq N$ such that

$$
D_{i} \cdot C_{j}= \begin{cases}1 & (i=j) \\ 0 & (i \neq j)\end{cases}
$$

by [12, Lemma 3.4.4]. Therefore we have

$$
N^{1}(X / Y)=\bigoplus_{1 \leq i \leq N} \mathbb{R}\left[D_{i}\right], \quad N_{1}(X / Y)=\bigoplus_{1 \leq i \leq N} \mathbb{R}\left[C_{i}\right]
$$

in our case. Similarly we introduce the one dimensional $\mathbb{R}$-vector spaces,

$$
N^{0}(X / Y)=\mathbb{R}[X], \quad N_{0}(X / Y)=\mathbb{R}[p],
$$

for a closed point $p \in C$ as numerical classes of codimension zero cycles and zero dimensional cycles. We have the pairing,

$$
N^{0}(X / Y) \times N_{0}(X / Y) \ni(a[X], b[p]) \longmapsto a b \in \mathbb{R} .
$$

Let $N^{1}(X / Y)_{\mathbb{C}}:=N^{1}(X / Y) \otimes_{\mathbb{R}} \mathbb{C}$.

Definition 3.2. We define the ample cone $A(X / Y)$ and the complexified ample cone $A(X / Y)_{\mathbb{C}}$ to be

$$
\begin{aligned}
A(X / Y) & :=\{\text { Numerical classes of ample } \mathbb{R} \text {-divisors }\} \subset N^{1}(X / Y) \\
& =\bigoplus_{1 \leq i \leq N} \mathbb{R}_{>0}\left[D_{i}\right] \\
A(X / Y)_{\mathbb{C}} & :=\left\{\beta+i \omega \in N^{1}(X / Y)_{\mathbb{C}} \mid \omega \in A(X / Y)\right\} .
\end{aligned}
$$

Also for each $1 \leq j \leq N$ and $k \in \mathbb{Z}$, define $\mathcal{W}_{j}$ and $\mathcal{W}_{j, k}$ to be

$$
\begin{aligned}
\mathcal{W}_{j} & :=\left\{\beta+i \omega \in N^{1}(X / Y)_{\mathbb{C}} \mid \omega \cdot C_{j}=0 \text { and } \omega \cdot C_{j^{\prime}}>0 \text { for } j \neq j^{\prime}\right\}, \\
\mathcal{W}_{j, k} & :=\left\{\beta+i \omega \in \mathcal{W}_{j} \mid \beta \cdot C_{j} \in(k-1, k)\right\} .
\end{aligned}
$$

Note that the union $\bigcup_{1 \leq j \leq N} \mathcal{W}_{j}$ is the codimension one boundary of the complexified nef cone $\bar{A}(X / Y)_{\mathbb{C}} \subset N^{1}(X / Y)_{\mathbb{C}}$. For each $1 \leq i \leq N$, some multiple of the divisor

$$
\sum_{j \neq i} D_{j}
$$

is base point free by [25, Theorem 3.3]; thus there exists a birational contraction $g_{i}: X \rightarrow Y_{i}$ which contracts only $C_{i}$. Then $\mathcal{W}_{i}$ is written as

$$
\mathcal{W}_{i}=g_{i}^{*} \mathcal{A}\left(Y_{i} / Y\right)_{\mathbb{C}}
$$


Furthermore one can construct its flop [25, Theorem 6.14] and obtain the diagram below:

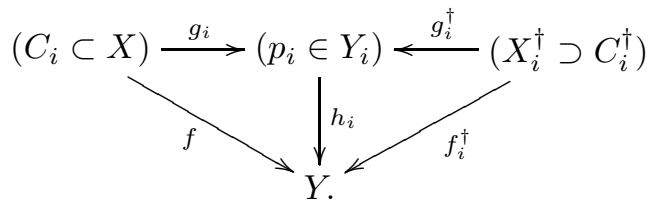

Next let $g: W \rightarrow Y$ be another crepant small resolution, and $\phi=f^{-1} \circ g: W \rightarrow X$ be the birational map. Because $\phi$ is an isomorphism in codimension one, one has the isomorphism of the groups of divisor classes, $\phi_{*}: N^{1}(W / Y) \rightarrow N^{1}(X / Y)$, called the strict transform. We use the same notation $\phi_{*}: \operatorname{Pic}(W) \rightarrow \operatorname{Pic}(X)$ for the isomorphism of Picard groups. The following theorem gives a chamber structure on $N^{1}(X / Y)$, where each chamber corresponds to the ample cone of a crepant small resolution of $Y$.

Theorem 3.3 ([23, Main theorem], [19, Theorem 2.3]). The number of crepant small resolutions $g: W \rightarrow Y$ is finite up to isomorphism. One has the decomposition,

$$
N^{1}(X / Y)=\bigcup_{(W, \phi)} \phi_{*} \bar{A}(W / Y)
$$

Here $(W, \phi)$ is a pair of a crepant small resolution $g: W \rightarrow Y$ and the birational map $\phi=f^{-1} \circ g$. Moreover $\phi_{*} A(W / Y) \cap \phi_{*}^{\prime} A\left(W^{\prime} / Y\right) \neq \emptyset$ if and only if there exists an isomorphism $h: W^{\prime} \rightarrow W$ such that $\phi \circ h=\phi^{\prime}$.

Remark 3.4. In our case, $f: X \rightarrow Y$ is an isomorphism in codimension one. Therefore the $f$-effective $f$-movable cone and $f$-effective $f$-nef cone defined in [19, Definition 1.1] coincide with $N^{1}(X / Y)$ and $\bar{A}(X / Y)$, respectively.

3.2. Chern characters and Riemann-Roch theorem. We consider the Chern characters which take values in our vector spaces $N^{*}(X / Y)$ and $N_{*}(X / Y)$,

$$
\begin{aligned}
& D^{b}(X) \ni E \longmapsto\left(\operatorname{ch}_{0}(E), \operatorname{ch}_{1}(E)\right) \in N^{0}(X / Y) \oplus N^{1}(X / Y), \\
& \mathcal{D}_{X / Y} \ni F \longmapsto\left(\operatorname{ch}_{2}(F), \operatorname{ch}_{3}(F)\right) \in N_{1}(X / Y) \oplus N_{0}(X / Y) .
\end{aligned}
$$

Usually, Chern characters take values in Chow groups. But since rationally equivalent two cycles are numerically equivalent, $\mathrm{ch}_{0}, \mathrm{ch}_{1}$ can take values in $N^{0}(X / Y)$, $N^{1}(X / Y)$. Also $F \in \mathcal{D}_{X / Y}$ is supported on $C$; thus $\mathrm{ch}_{2}, \mathrm{ch}_{1}$ take values in $N_{1}(X / Y), N_{0}(X / Y)$.

Let us take $E \in D^{b}(X), F \in \mathcal{D}_{X / Y}$. Since the support of $F$ is proper, the space $\operatorname{Ext}_{X}^{i}(E, F)$ is finite dimensional, and zero except for a finite number of $i \in \mathbb{Z}$. Therefore the number

$$
\chi(E, F):=\sum_{i \in \mathbb{Z}}(-1)^{i} \operatorname{dim} \operatorname{Ext}_{X}^{i}(E, F) \in \mathbb{Z}
$$

makes sense. The Riemann-Roch theorem [15, Corollary 18.3.1] implies

$$
\chi(E, F)=\operatorname{ch}_{0}(E) \cdot \operatorname{ch}_{3}(F)-\operatorname{ch}_{1}(E) \cdot \operatorname{ch}_{2}(F) .
$$


3.3. Perverse t-structures. Here we introduce the Abelian categories ${ }^{p} \operatorname{Per}(X / Y)$ $\subset D^{b}(X)$ for $p=0,-1$, which were introduced in [6] to construct the flops. These categories provide a central technical tool in this paper. For the precise definition, we refer to [6, Section 3] and [12, Section 3]. First we give the known results of [6], [12. Let $S_{0}, S_{0}^{\prime}$, and $S_{i}$ for $1 \leq i \leq N$ be

$$
S_{0}:=\omega_{C}[1], \quad S_{0}^{\prime}:=\mathcal{O}_{C}, \quad S_{i}:=\mathcal{O}_{C_{i}}(-1) .
$$

Here the scheme structure on $C_{i}$ is reduced in the definition of $S_{i}$ for $1 \leq i \leq N$. However the scheme structure on $C$ in the definition of $S_{0}, S_{0}^{\prime}$ must be the scheme theoretic fiber of $f: X \rightarrow Y$, thus possibly non-reduced.

Lemma 3.5 ([6, Lemma 3.2], [12, Section 3]). For $p=0$ or -1 , there exist hearts of bounded t-structures ${ }^{p} \operatorname{Per}(X / Y) \subset D^{b}(X)$, such that $E \in D^{b}(X)$ belongs to ${ }^{p} \operatorname{Per}(X / Y)$ if and only if the following holds:

(i) $E$ is concentrated in degrees $[-1,0]$.

(ii) $f_{*} H^{-1}(E)=0$ and $R^{1} f_{*} H^{0}(E)=0$.

(iii) For $j \geq 1$, we have the following:

$$
\operatorname{Hom}\left(S_{j}, H^{-1}(E)\right)=0 \quad(p=0), \quad \operatorname{Hom}\left(H^{0}(E), S_{j}\right)=0 \quad(p=-1) .
$$

Here we define the categories ${ }^{p} \operatorname{Per}\left(\mathcal{D}_{X / Y}\right)$ for $p=-1,0$ to be

$$
{ }^{p} \operatorname{Per}\left(\mathcal{D}_{X / Y}\right):={ }^{p} \operatorname{Per}(X / Y) \cap \mathcal{D}_{X / Y} .
$$

M. Van den Bergh 12 determined the simple objects of these categories.

Proposition 3.6 ([12, Proposition 3.5.8]). The categories

$$
{ }^{0} \operatorname{Per}\left(\mathcal{D}_{X / Y}\right), \quad \text { and } \quad{ }^{-1} \operatorname{Per}\left(\mathcal{D}_{X / Y}\right)
$$

are hearts of bounded $t$-structures on $\mathcal{D}_{X / Y}$ and are finite length Abelian categories. Their simple objects are

$$
\left\{S_{i}\right\}_{0 \leq i \leq N} \text { and }\left\{S_{0}^{\prime}, S_{i}[1]\right\}_{1 \leq i \leq N},
$$

respectively.

Let $g_{i}: X \rightarrow Y_{i}$ be the contraction which contracts $C_{i}$, as in the diagram (1). Note that one can define ${ }^{p} \operatorname{Per}\left(X / Y_{i}\right) \subset D^{b}(X)$ and ${ }^{p} \operatorname{Per}\left(\mathcal{D}_{X / Y_{i}}\right) \subset \mathcal{D}_{X / Y_{i}}$ similarly. We introduce the following subcategories of $\mathcal{D}_{X / Y}$.

Definition 3.7. For $1 \leq i \leq N$ and $k \in \mathbb{Z}$, we define $\mathcal{A}_{(i, k)}$ by

$$
\mathcal{A}_{(i, k)}:=\left({ }^{0} \operatorname{Per}\left(X / Y_{i}\right) \cap \mathcal{D}_{X / Y}\right) \otimes \mathcal{O}_{X}\left(k D_{i}\right) .
$$

The following lemma will be used in the next section. We give the proof in Section 6 .

Lemma 3.8. (i) For $p=-1,0$, we have

$$
\operatorname{Coh}_{\cup_{j \neq i} C_{j}}(X) \cup{ }^{p} \operatorname{Per}\left(\mathcal{D}_{X / Y_{i}}\right) \subset{ }^{p} \operatorname{Per}\left(X / Y_{i}\right) \cap \mathcal{D}_{X / Y}
$$

as subcategories of $\mathcal{D}_{X / Y}$. Moreover the right hand side coincides with the smallest extension closed subcategory of $\mathcal{D}_{X / Y}$ which contains the left hand side.

(ii) We have the following equality of subcategories of $\mathcal{D}_{X / Y}$ :

$$
{ }^{-1} \operatorname{Per}\left(X / Y_{i}\right) \cap \mathcal{D}_{X / Y}=\mathcal{A}_{(i, 1)}
$$


(iii) Any object in $\mathcal{A}_{(i, k)}$ is given by a successive extension of the objects in

$$
\mathrm{Coh}_{\cup_{j \neq i} C_{j}}(X), \quad \mathcal{O}_{C_{i}}(k-2)[1], \quad \mathcal{O}_{C_{i}}(k-1) .
$$

Here $C_{i}$ has a reduced scheme structure.

Remark 3.9. As we see in Section 6, the assumption that $X_{0}$ is smooth is needed in the proof of Lemma 3.8 (ii), (iii). If otherwise $C_{i}$ in Lemma 3.8 (iii) may not be reduced, so the arguments in the next section (especially Lemma 4.3) do not work.

3.4. Fourier-Mukai transforms and standard equivalences. The FourierMukai transform is a useful tool in studying derived categories. Let $g: W \rightarrow Y$ be another crepant resolution. We introduce the relative version of the Fourier-Mukai transform.

Definition 3.10. We say the equivalence $\Phi: D^{b}(W) \rightarrow D^{b}(X)$ is of Fourier-Mukai type over $Y$ if there exists an object $\mathcal{E} \in D^{b}(X \times W)$, which is supported on $X \times_{Y} W$, such that $\Phi$ is written as

$$
\Phi \cong \Phi_{W \rightarrow X}^{\mathcal{E}}:=\mathbf{R} p_{X *}\left(p_{W}^{*}(*) \stackrel{\mathbf{L}}{\otimes} \mathcal{E}\right) .
$$

Here $p_{X}, p_{W}$ are corresponding projections from $X \times W$. The object $\mathcal{E} \in D^{b}(X \times W)$ is called the kernel of $\Phi$.

The following theorem plays an important role in this paper.

Theorem 3.11 ([6, Theorem 1.1], [11, Proposition 4.2]). Let $g: W \rightarrow Y$ be another crepant small resolution, and $\phi: W \rightarrow X$ be the birational map. Assume for a $g$ ample divisor $H$ on $W$, the divisor $-\phi_{*} H$ is $f$-ample. Then the functor

$$
\Phi_{W \rightarrow X}^{\mathcal{O}_{W \times Y^{X}}}: D^{b}(W) \longrightarrow D^{b}(X)
$$

gives an equivalence and takes ${ }^{-1} \operatorname{Per}(W / Y)$ to ${ }^{0} \operatorname{Per}(X / Y)$.

We call the equivalence given in Theorem 3.11 standard.

Remark 3.12. Let us apply Theorem 3.11 to the sequence $X_{i}^{\dagger} \rightarrow Y_{i} \leftarrow X$ as in the diagram (1). Then there exists a standard equivalence

$$
D^{b}\left(X_{i}^{\dagger}\right) \longrightarrow D^{b}(X)
$$

which takes ${ }^{-1} \operatorname{Per}\left(X_{i}^{\dagger} / Y_{i}\right)$ to ${ }^{0} \operatorname{Per}\left(X / Y_{i}\right)$ but not necessarily takes ${ }^{-1} \operatorname{Per}\left(X_{i}^{\dagger} / Y\right)$ to ${ }^{0} \operatorname{Per}(X / Y)$.

We introduce the set $\operatorname{FM}(X)$ as follows.

Definition 3.13. We define $\operatorname{FM}(X)$ to be the set of pairs $(W, \Phi)$, where $g: W \rightarrow Y$ is another crepant small resolution and $\Phi: D^{b}(W) \rightarrow D^{b}(X)$ is an equivalence of derived categories which satisfies the following: there exists a factorization of the birational map $\phi: W \rightarrow X$,

$$
W=X^{n} \rightarrow X^{n-1} \rightarrow \cdots \rightarrow X^{1} \rightarrow X^{0}=X,
$$

and equivalences of Fourier-Mukai type over $Y, \Phi^{j}: D^{b}\left(X^{j}\right) \rightarrow D^{b}\left(X^{j-1}\right)$ such that $\Phi \cong \Phi^{1} \circ \cdots \circ \Phi^{n}$. Each $\Phi^{j}$ is one of the following:

- type $I: X^{j}=X^{j-1}$ and $\Phi^{j} \cong \otimes \mathcal{L}$ for $\mathcal{L} \in \operatorname{Pic}\left(X^{j}\right)$.

- type II : $X^{j} \rightarrow X^{j-1}$ is a flop at a single rational curve as in the diagram $(1)$, and $\Phi^{j}$ is a standard equivalence. 
Note that for $(W, \Phi) \in \mathrm{FM}(X), \Phi$ restricts to the equivalence,

$$
\Phi: \mathcal{D}_{W / Y} \longrightarrow \mathcal{D}_{X / Y},
$$

because the kernel of $\Phi$ is supported on $X \times_{Y} W$. Therefore $\Phi$ induces an isomorphism between $K\left(\mathcal{D}_{W / Y}\right)$ and $K\left(\mathcal{D}_{X / Y}\right)$. For the description of $K\left(\mathcal{D}_{X / Y}\right)$, we have the following lemma. We give the proof in Section 6 .

Lemma 3.14. (i) The class $\left[\mathcal{O}_{x}\right] \in K\left(\mathcal{D}_{X / Y}\right)$ does not depend on a choice of $x \in C$, and $K\left(\mathcal{D}_{X / Y}\right)$ is described by the direct sum,

$$
K\left(\mathcal{D}_{X / Y}\right)=\mathbb{Z}\left[\mathcal{O}_{x}\right] \oplus \bigoplus_{i=1}^{N} \mathbb{Z}\left[\mathcal{O}_{C_{i}}(-1)\right] .
$$

(ii) For $(W, \Phi) \in \operatorname{FM}(X)$, $\Phi$ takes the class $\left[\mathcal{O}_{w}\right] \in K\left(\mathcal{D}_{W / Y}\right)$ to $\left[\mathcal{O}_{x}\right] \in$ $K\left(\mathcal{D}_{X / Y}\right)$ for closed points $w \in W$ and $x \in X$.

Finally we give the following lemma, which relates Fourier-Mukai transforms to Chern characters. The proof will also be given in Section 6 .

Lemma 3.15. For $(W, \Phi) \in \operatorname{FM}(X)$ and $\mathcal{L} \in \operatorname{Pic}(W)$, one has

$$
\operatorname{ch}_{1} \Phi(\mathcal{L})=\phi_{*} \operatorname{ch}_{1} \mathcal{L}+\operatorname{ch}_{1} \Phi\left(\mathcal{O}_{W}\right) .
$$

Here $\phi: W \rightarrow X$ is the birational map.

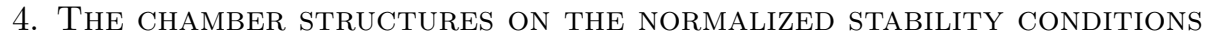

In this section, we prove Theorem 1.1. As in the introduction, let $\operatorname{Stab}(X / Y)$ be the space of stability conditions on $\mathcal{D}_{X / Y}$.

4.1. Constructions of stability conditions. Here we construct stability conditions that correspond to a neighborhood of the large volume limit. By Proposition 2.2 in order to give a stability condition, we find a heart of a bounded t-structure $\mathcal{A} \subset \mathcal{D}_{X / Y}$ and a stability function $Z: K\left(\mathcal{D}_{X / Y}\right) \rightarrow \mathbb{C}$. As a heart of a bounded t-structure, we take the subcategory

$$
\operatorname{Coh}_{C}(X)=\operatorname{Coh}(X) \cap \mathcal{D}_{X / Y} \subset \mathcal{D}_{X / Y}
$$

In order to construct stability functions, let us take an element $\beta+i \omega \in N^{1}(X / Y)_{\mathbb{C}}$. We define $Z_{(\beta, \omega)}: K\left(\mathcal{D}_{X / Y}\right) \rightarrow \mathbb{C}$ as follows:

$$
\begin{aligned}
Z_{(\beta, \omega)}(E) & :=-\int e^{-(\beta+i \omega)} \operatorname{ch}(E) \\
& =-\operatorname{ch}_{3}(E)+(\beta+i \omega) \operatorname{ch}_{2}(E) .
\end{aligned}
$$

Lemma 4.1. Assume $\beta+i \omega \in A(X / Y)_{\mathbb{C}}$. Then the pair

$$
\sigma_{(\beta, \omega)}:=\left(Z_{(\beta, \omega)}, \operatorname{Coh}_{C}(X)\right)
$$

determines a point of $\operatorname{Stab}(X / Y)$.

Proof. We use Proposition 2.2 and Remark 2.3 . Let us take a non-zero object $E \in \operatorname{Coh}_{C}(X)$. If $\operatorname{dim} \operatorname{Supp}(E)=1$, then $\operatorname{Im} Z_{(\beta, \omega)}(E)>0$ since $\omega$ is ample. If $\operatorname{dim} \operatorname{Supp}(E)=0$, then $Z_{(\beta, \omega)}(E) \in \mathbb{R}_{<0}$. Therefore it suffices to show the HarderNarasimhan property. 
By Remark 2.3. we check that $\operatorname{Coh}_{C}(X)$ is noetherian. But this is obvious, because a finitely generated module over a noetherian ring is noetherian. Thus it is enough to check that there is no infinite sequence,

$$
\cdots \subset E_{j+1} \subset E_{j} \subset \cdots \subset E_{2} \subset E_{1},
$$

for $E_{j} \in \operatorname{Coh}_{C}(X)$ with $\phi\left(E_{j+1}\right)>\phi\left(E_{j}\right)$ for all $j$. Assume the sequence as above exists. For $1 \leq i \leq N$, let $\eta_{i} \in X$ be the generic point of $C_{i}$. Then since the $E_{j}$ are supported on $C$, one has

$$
0 \leq \text { length }_{\mathcal{O}_{X, \eta_{i}}} E_{j+1} \leq \operatorname{length}_{\mathcal{O}_{X, \eta_{i}}} E_{j}<\infty
$$

Therefore we may assume length $\mathcal{O}_{X, \eta_{i}} E_{j}$ is constant for all $i$ and $j$. Then if we take the exact sequence,

$$
0 \longrightarrow E_{j+1} \rightarrow E_{j} \rightarrow G_{j} \rightarrow 0,
$$

then $G_{j}$ is zero dimensional; thus $Z_{(\beta, \omega)}\left(G_{j}\right) \in \mathbb{R}_{\leq 0}$. This implies $\phi\left(E_{j}\right) \geq \phi\left(E_{j+1}\right)$; thus we get a contradiction.

The stability conditions $\sigma_{(\beta, \omega)}$ are contained in the following subspace:

$$
\operatorname{Stab}_{\mathrm{n}}(X / Y):=\left\{\sigma=(Z, \mathcal{P}) \in \operatorname{Stab}(X / Y) \mid Z\left(\left[\mathcal{O}_{x}\right]\right)=-1\right\},
$$

for closed points $x \in C$. We call $\operatorname{Stab}_{\mathrm{n}}(X / Y)$ the set of normalized stability conditions. By Lemma 3.14 (i), the following map gives an isomorphism of the vector spaces:

$$
N^{1}(X / Y)_{\mathbb{C}} \ni \beta+i \omega \longmapsto Z_{(\beta, \omega)} \in\left\{Z \in \operatorname{Hom}\left(K\left(\mathcal{D}_{X / Y}\right), \mathbb{C}\right) \mid Z\left(\left[\mathcal{O}_{x}\right]\right)=-1\right\} .
$$

Hence the map sending stability conditions to their central charges restricts to give the map

$$
\mathcal{Z}_{X}: \operatorname{Stab}_{\mathrm{n}}(X / Y) \longrightarrow N^{1}(X / Y)_{\mathbb{C}}
$$

We give the following definition.

Definition 4.2. We define $U_{X} \subset \operatorname{Stab}_{\mathrm{n}}(X / Y)$ as follows:

$$
U_{X}:=\left\{\sigma_{(\beta, \omega)} \in \operatorname{Stab}_{\mathrm{n}}(X / Y) \mid \beta+i \omega \in A(X / Y)_{\mathbb{C}}\right\} .
$$

Also define

$$
\operatorname{Stab}_{\mathrm{n}}^{\circ}(X / Y) \subset \operatorname{Stab}_{\mathrm{n}}(X / Y)
$$

to be the connected component of $\operatorname{Stab}_{\mathrm{n}}(X / Y)$ which contains $U_{X}$.

Note that $\mathcal{Z}_{X}$ restricts to a homeomorphism between $U_{X}$ and $A(X / Y)_{\mathbb{C}}$.

4.2. Codimension one boundaries of $U_{X}$. Here we give the descriptions of codimension one boundaries of the domain $U_{X}$. Let us take a point,

$$
\sigma=\left(Z_{(\beta, \omega)}, \mathcal{A}\right) \in \bar{U}_{X}
$$

Note that by the continuity of $\mathcal{Z}_{X}$, we have

$$
\beta+i \omega=\mathcal{Z}_{X}(\sigma) \in \bar{A}(X / Y)_{\mathbb{C}}
$$

We say $\sigma$ lies in the codimension one boundary if $\mathcal{Z}_{X}(\sigma) \in \mathcal{W}_{i}$ for some $1 \leq i \leq N$. The purpose here is to find a heart of a t-structure $\mathcal{A}$ such that the pair $\left(Z_{(\beta, \omega)}, \mathcal{A}\right)$ gives a stability condition and lies in the codimension one boundary. Recall that we defined $\mathcal{W}_{i, k}$ in Definition 3.2 and $\mathcal{A}_{(i, k)}$ in Definition 3.7. 
Lemma 4.3. Let us take $\beta+i \omega \in \mathcal{W}_{i, k}$ for some $k \in \mathbb{Z}$. Then the pair

$$
\sigma_{(\beta, \omega)}:=\left(Z_{(\beta, \omega)}, \mathcal{A}_{(i, k)}\right)
$$

gives a stability condition on $\mathcal{D}_{X / Y}$.

Proof. First we check the following:

$$
Z_{(\beta, \omega)}\left(\mathcal{A}_{(i, k)}\right) \subset\left\{\mathbb{R}_{>0} \exp (i \pi \phi) \mid 0<\phi \leq 1\right\} .
$$

By Lemma 3.8 (iii), $\mathcal{A}_{(i, k)}$ is the smallest extension closed subcategory of $\mathcal{D}_{X / Y}$ which contains

$$
\operatorname{Coh}_{\cup_{j \neq i} C_{j}}(X), \quad \mathcal{O}_{C_{i}}(k-2)[1], \quad \mathcal{O}_{C_{i}}(k-1) .
$$

Hence it is enough to check (2) for the above objects. Let us take $E \in \operatorname{Coh}_{\cup_{j \neq i} C_{j}}(X)$. If $\operatorname{dim} \operatorname{Supp}(E)=1$, then $\operatorname{Im} Z(E)>0$ because $\omega \cdot C_{j}>0$ for $j \neq i$. If $\operatorname{dim} \operatorname{Supp}(E)$ $=0$, one has $Z_{(\beta, \omega)}(E) \in \mathbb{R}_{<0}$. Therefore (2) holds for $E \in \mathrm{Coh}_{\cup_{j \neq i} C_{j}}(X)$. For the other two objects, one has

$$
\begin{aligned}
& Z_{(\beta, \omega)}\left(\mathcal{O}_{C_{i}}(k-2)[1]\right)=k-1-\beta \cdot C_{i} \in \mathbb{R}_{<0}, \\
& Z_{(\beta, \omega)}\left(\mathcal{O}_{C_{i}}(k-1)\right)=-k+\beta \cdot C_{i} \in \mathbb{R}_{<0} .
\end{aligned}
$$

Therefore (2) holds, and it is enough to check that $\mathcal{A}_{(i, k)}$ is noetherian and that the pair $\left(Z_{(\beta, \omega)}, \mathcal{A}_{(i, k)}\right)$ satisfies the Harder-Narasimhan property, by Remark 2.3.

First we check that $\mathcal{A}_{(i, k)}$ is noetherian. Assume there exists an infinite sequence

$$
E_{1} \subset E_{2} \subset \cdots E_{n} \subset \cdots \subset E
$$

in $\mathcal{A}_{(i, k)}$. We put $E_{\infty}=E$. By Lemma 3.8 (iii), the complex $E_{n}$ is concentrated in degree zero at the generic point of $C_{j}$ for $j \neq i$. Hence we may assume the generic lengths of $E_{n}$ at $C_{j}$ are constant for all $j \neq i$. Then let us take the exact sequence

$$
0 \rightarrow E_{1} \rightarrow E_{n} \rightarrow G_{n} \rightarrow 0
$$

in $\mathcal{A}_{(i, k)}$. Then $G_{n}$ is supported on $C_{i}$; hence

$$
G_{n} \in{ }^{0} \operatorname{Per}\left(\mathcal{D}_{X / Y_{i}}\right) \otimes \mathcal{O}_{X}\left(k D_{i}\right) .
$$

We obtain the sequence

$$
G_{1} \subset G_{2} \subset \cdots \subset G_{\infty}
$$

in ${ }^{0} \operatorname{Per}\left(\mathcal{D}_{X / Y_{i}}\right) \otimes \mathcal{O}_{X}\left(k D_{i}\right)$. By Proposition 3.6, ${ }^{0} \operatorname{Per}\left(\mathcal{D}_{X / Y_{i}}\right) \otimes \mathcal{O}_{X}\left(k D_{i}\right)$ is of finite length; hence this sequence terminates.

Finally we check the Harder-Narasimhan property. Let us assume there exists an infinite sequence

$$
\cdots \subset E_{n} \subset \cdots \subset E_{2} \subset E_{1}
$$

in $\mathcal{A}_{(i, k)}$ such that $\phi\left(E_{n+1}\right)>\phi\left(E_{n}\right)$ for all $n$. Then $\operatorname{Im} Z_{(\beta, \omega)}\left(E_{n}\right)>0$. Again we may assume the generic lengths of $E_{n}$ at $C_{j}$ are constant for $j \neq i$. If we take the exact sequence

$$
0 \rightarrow E_{n+1} \rightarrow E_{n} \rightarrow G_{n} \rightarrow 0
$$

in $\mathcal{A}_{(i, k)}$, then $G_{n}$ is supported on $C_{i}$; hence $Z_{(\beta, \omega)}\left(G_{n}\right) \in \mathbb{R}_{\leq 0}$. But this implies $\phi\left(E_{n}\right) \geq\left(E_{n+1}\right)$, hence a contradiction.

The following proposition gives the complete description of the codimension one boundaries of $U_{X}$. 
Proposition 4.4. For $\beta+i \omega \in \mathcal{W}_{i}$, there exists a point $\sigma \in \bar{U}_{X}$ with $\mathcal{Z}_{X}(\sigma)=$ $\beta+i \omega$ if and only if $\beta+i \omega \in \mathcal{W}_{i, k}$ for some $k \in \mathbb{Z}$. If $\beta+i \omega \in \mathcal{W}_{i, k}$ for $k \in \mathbb{Z}$, the point

$$
\sigma_{(\beta, \omega)}=\left(Z_{(\beta, \omega)}, \mathcal{A}_{(i, k)}\right) \in \operatorname{Stab}_{\mathrm{n}}(X / Y)
$$

in Lemma 4.3 gives the point of $\bar{U}_{X}$.

Proof. First assume there exists $\sigma \in \bar{U}_{X}$ with $\mathcal{Z}_{X}(\sigma)=\beta+i \omega \in \mathcal{W}_{i}$. It is easy to see that $\mathcal{O}_{C_{i}}(k-1)$ is stable with respect to all $\sigma^{\prime} \in U_{X}$ and $k \in \mathbb{Z}$. By the comment in [5] after [5. Proposition 8.1], $\mathcal{O}_{C_{i}}(k-1)$ is at least semistable in $\sigma$. This implies

$$
Z_{(\beta, \omega)}\left(\mathcal{O}_{C_{i}}(k-1)\right)=-k+\beta \cdot C_{i} \neq 0,
$$

for all $k \in \mathbb{Z}$. Therefore we have $\beta \cdot C_{i} \notin \mathbb{Z}$.

Conversely assume that $\beta \cdot C_{i} \in(k-1, k)$ for some $k \in \mathbb{Z}$. In this case, we have the stability condition

$$
\sigma:=\sigma_{(\beta, \omega)}=\left(Z_{(\beta, \omega)}, \mathcal{A}_{(i, k)}\right),
$$

constructed in Lemma 4.3. Therefore it is enough to check $\sigma \in \bar{U}_{X}$. We leave it to the next lemma, and give the proof in Section 6, because it requires some technical arguments.

Lemma 4.5. $\sigma_{(\beta, \omega)}=\left(Z_{(\beta, \omega)}, \mathcal{A}_{(i, k)}\right)$, constructed in Lemma 4.3, is contained in $\bar{U}_{X}$.

Now we give the following definition.

Definition 4.6. We define $\partial U_{X}(i, k)$ for $1 \leq i \leq N$ and $k \in \mathbb{Z}$ by

$$
\partial U_{X}(i, k)=\left\{\left(Z_{(\beta, \omega)}, \mathcal{A}_{(i, k)}\right) \in \bar{U}_{X} \mid \beta+i \omega \in \mathcal{W}_{i, k}\right\} .
$$

4.3. Other regions by Fourier-Mukai transforms. Here we construct other regions using $\operatorname{FM}(X)$ defined in Definition 3.13, Let us take $u=(W, \Phi) \in \operatorname{FM}(X)$. Recall that $\Phi$ gives an equivalence $\Phi: \mathcal{D}_{W / Y} \rightarrow \mathcal{D}_{X / Y}$. Then for any $\sigma=(Z, \mathcal{P}) \in$ $\operatorname{Stab}(W / Y)$, one gets the stability condition $\Phi_{*} \sigma=\left(Z^{\prime}, \mathcal{P}^{\prime}\right)$ by

$$
Z^{\prime}(E):=Z \circ \Phi^{-1}(E), \quad \mathcal{P}^{\prime}(E):=\Phi \mathcal{P}(\phi),
$$

for $E \in \mathcal{D}_{X / Y}$ and $\phi \in \mathbb{R}$. Obviously, the map

$$
\Phi_{*}: \operatorname{Stab}(W / Y) \longrightarrow \operatorname{Stab}(X / Y)
$$

gives a homeomorphism. Furthermore by Lemma 3.14 (ii), $\Phi_{*}$ restricts to the homeomorphism

$$
\Phi_{*}: \operatorname{Stab}_{\mathrm{n}}(W / Y) \longrightarrow \operatorname{Stab}_{\mathrm{n}}(X / Y) .
$$

Definition 4.7. For $u=(W, \Phi) \in \operatorname{FM}(X)$, we define the open set $U(W, \Phi)$ in $\operatorname{Stab}_{\mathrm{n}}(X / Y)$ to be

$$
U(W, \Phi):=\Phi_{*} U_{W} \subset \operatorname{Stab}_{\mathrm{n}}(X / Y),
$$

and the map $\phi^{u}: N^{1}(W / Y)_{\mathbb{C}} \rightarrow N^{1}(X / Y)_{\mathbb{C}}$ to be

$$
\phi^{u}(D)=\operatorname{ch}_{1} \Phi\left(\mathcal{O}_{W}\right)+\phi_{*} D .
$$

Here $\phi$ is the birational map $\phi: W \rightarrow X$.

We have introduced the map $\phi^{u}$ because the following lemma holds. 
Lemma 4.8. For $u=(W, \Phi) \in \operatorname{FM}(X)$, we have the following commutative diagram:

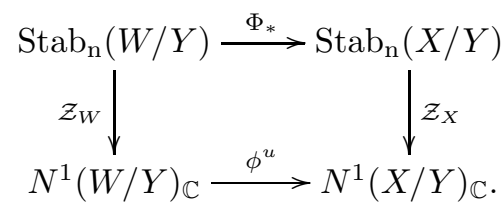

Proof. Let us take $\sigma=\left(Z_{(\beta, \omega)}, \mathcal{P}\right) \in \operatorname{Stab}_{\mathrm{n}}(W / Y)$, and $\mathcal{L} \in \operatorname{Pic}(W)_{\mathbb{C}}$ with $\operatorname{ch}_{1}(\mathcal{L})=$ $\beta+i \omega$. Then for $F \in \mathcal{D}_{W / Y}$, the value $Z_{(\beta, \omega)}(F)$ is written as $-\chi(\mathcal{L}, F)$ by the Riemann-Roch theorem. Therefore for $E \in \mathcal{D}_{X / Y}$, one calculates $Z_{(\beta, \omega)} \Phi^{-1}(E)$ as follows:

$$
\begin{aligned}
Z_{(\beta, \omega)} \Phi^{-1}(E) & =-\chi\left(\mathcal{L}, \Phi^{-1}(E)\right) \\
& =-\chi(\Phi(\mathcal{L}), E) \\
& =-\operatorname{ch}_{0} \Phi(\mathcal{L}) \operatorname{ch}_{3} E+\operatorname{ch}_{1} \Phi(\mathcal{L}) \operatorname{ch}_{2} E \\
& =-\operatorname{ch}_{3} E+\left(\phi_{*} \operatorname{ch}_{1} \mathcal{L}+\operatorname{ch}_{1} \Phi\left(\mathcal{O}_{W}\right)\right) \operatorname{ch}_{2} E \\
& =-\operatorname{ch}_{3} E+\phi^{u}(\beta+i \omega) \operatorname{ch}_{2} E .
\end{aligned}
$$

Here the fourth isomorphism follows from Lemma 3.15. The above equality exactly means $\mathcal{Z}_{X} \circ \Phi_{*}=\phi^{v} \circ \mathcal{Z}_{W}$.

We have the following corollary.

Corollary 4.9. $\mathcal{Z}_{X}$ restricts to give a homeomorphism between $U(W, \Phi)$ and $\phi_{*} A(W / Y)_{\mathbb{C}}$.

4.4. Chambers in $\operatorname{Stab}_{\mathbf{n}}(X / Y)$. Here we investigate the relationship of the regions $U(W, \Phi)$.

Proposition 4.10. The regions $U(W, \Phi)$ satisfy the following:

(i) $U(W, \Phi) \cap U\left(W^{\prime}, \Phi^{\prime}\right) \neq \emptyset$ if and only if there exists $\mathcal{L} \in \operatorname{Pic}(W)$ such that

$$
W \cong W^{\prime} \quad \text { and } \quad \Phi^{-1} \circ \Phi^{\prime} \cong \otimes \mathcal{L} .
$$

(ii) $U(W, \Phi) \cap U\left(W^{\prime}, \Phi^{\prime}\right)=\emptyset$ but $\bar{U}(W, \Phi) \cap \bar{U}\left(W^{\prime}, \Phi^{\prime}\right) \neq \emptyset$ in a codimension one wall if and only if $W \rightarrow W^{\prime}$ is a flop at a single rational curve, and we have

$$
\Phi^{\prime}-1 \circ \Phi \cong \otimes \mathcal{L}^{\prime} \circ \Phi^{\prime \prime} \circ \otimes \mathcal{L}
$$

with $\Phi^{\prime \prime}$ a standard equivalence

$$
\Phi^{\prime \prime}: D^{b}(W) \longrightarrow D^{b}\left(W^{\prime}\right)
$$

and $\mathcal{L} \in \operatorname{Pic}(W), \mathcal{L}^{\prime} \in \operatorname{Pic}\left(W^{\prime}\right)$. Moreover all points in a codimension one wall of $\bar{U}(W, \Phi)$ are contained in $\bar{U}\left(W^{\prime}, \Phi^{\prime}\right)$ with $\left(W^{\prime}, \Phi^{\prime}\right)$ as above.

Proof. (i) We may assume $W^{\prime}=X$ and $\Phi^{\prime}=$ id. Suppose $U(W, \Phi) \cap U(X$, id $) \neq \emptyset$. Then $\Phi$ takes $\operatorname{Coh}_{C}(W)$ to $\operatorname{Coh}_{C}(X)$. Note that simple objects of these categories are skyscraper sheaves. Hence for $p \in W$, we have $\Phi\left(\mathcal{O}_{p}\right) \cong \mathcal{O}_{\phi(p)}$ for some $\phi(p) \in$ $X$. Since $\Phi$ is of Fourier-Mukai type, $\phi$ gives an isomorphism $W \cong X$ and $\Phi \cong \otimes \mathcal{L}$ for some $\mathcal{L} \in \operatorname{Pic}(X)$ (cf. 21, Theorem 3.2]).

(ii) Again we may assume $W^{\prime}=X$ and $\Phi^{\prime}=$ id. First let us consider a flop $\phi_{i}: X_{i}^{\dagger} \rightarrow X$ as in the diagram (1). Let us consider the standard equivalence,

$$
\Phi_{i}: D^{b}\left(X_{i}^{\dagger}\right) \longrightarrow D^{b}(X)
$$


Then recall that $\Phi_{i}$ takes ${ }^{-1} \operatorname{Per}\left(X_{i}^{\dagger} / Y_{i}\right)$ to ${ }^{0} \operatorname{Per}\left(X / Y_{i}\right)$, by Theorem 3.11, Combined with Lemma 3.8 (ii), $\Phi_{i}$ restricts to give the equivalence:

$$
\Phi_{i}:\left({ }^{0} \operatorname{Per}\left(X_{i}^{\dagger} / Y_{i}\right) \cap \mathcal{D}_{X_{i}^{\dagger} / Y}\right) \otimes \mathcal{O}_{X_{i}^{\dagger}}\left(D_{i}^{\dagger}\right) \longrightarrow{ }^{0} \operatorname{Per}\left(X / Y_{i}\right) \cap \mathcal{D}_{X / Y} .
$$

Here $D_{i}^{\dagger}$ is a divisor on $X_{i}^{\dagger}$ with $D_{i}^{\dagger} \cdot C_{i}^{\dagger}=1$, and $D_{i}^{\dagger} \cdot C_{j}^{\dagger}=0$ for other exceptional curves $C_{j}^{\dagger}, j \neq i$.

By Lemma 4.4, both sides appear as t-structures of some points in codimension one boundaries of $U_{X_{i}^{\dagger}}$ and $U_{X}$, respectively. On the other hand if $\beta \in N^{1}\left(X_{i}^{\dagger} / Y\right)$ satisfies $\beta \cdot C_{i}^{\dagger} \in(0,1)$, then $\phi_{i *} \beta \cdot C_{i} \in(-1,0)$. Therefore Lemma 4.4 together with Lemma 4.8 gives the homeomorphism

$$
\Phi_{i *}: \partial U_{X_{i}^{\dagger}}(i, 1) \longrightarrow \partial U_{X}(i, 0) .
$$

Here recall that $\partial U_{X}(i, k)$ is defined in Definition 4.6. Similarly, we have a homeomorphism

$$
\left(\otimes \mathcal{O}_{X}\left(D_{i}\right)\right)_{*}: \partial U_{X}(i, k) \longrightarrow \partial U_{X}(i, k+1) .
$$

Combining these, for $\mathcal{L} \in \operatorname{Pic}\left(X_{i}^{\dagger}\right)$ and $\mathcal{L}^{\prime} \in \operatorname{Pic}(X)$ there exists a homeomorphism

$$
\left(\otimes \mathcal{L}^{\prime} \circ \Phi_{i} \circ \otimes \mathcal{L}\right)_{*}: \partial U_{X_{i}^{\dagger}}(i, l) \longrightarrow \partial U_{X}(i, k),
$$

for some $k$ and $l$, and conversely for all $k \in \mathbb{Z}$, there exist $\mathcal{L} \in \operatorname{Pic}\left(X_{i}^{\dagger}\right)$ and $\mathcal{L}^{\prime} \in \operatorname{Pic}(X)$ which gives the homeomorphism as above. This gives the "if" part and the last statement.

Conversely, assume that $\bar{U}(W, \Phi)$ and $\bar{U}_{X}$ intersect at a codimension one boundary, but $U(W, \Phi) \cap U_{X}=\emptyset$. Then, because $\operatorname{Stab}_{\mathrm{n}}(X / Y)$ is a complex manifold, there exists a pair $\left(X_{i}^{\dagger}, \Phi^{\dagger}\right)$ with $\Phi^{\dagger}=\otimes \mathcal{L}^{\prime} \circ \Phi_{i} \circ \otimes \mathcal{L}$ given as above, such that

$$
U(W, \Phi) \cap U\left(X_{i}^{\dagger}, \Phi^{\dagger}\right) \neq \emptyset \text {. }
$$

Then (i) implies $W \cong X_{i}^{\dagger}$ and $\Phi^{\dagger}$ is given as $\otimes \mathcal{L}^{\prime} \circ \Phi_{i} \circ \otimes \mathcal{L}^{\prime \prime}$ for some $\mathcal{L}^{\prime \prime} \in$ $\operatorname{Pic}(W)$.

4.5. Proof of Theorem 1.1. Now we give the proof of Theorem 1.1 .

Theorem 4.11. We have a disjoint union of chambers:

$$
\mathcal{M}:=\bigcup_{(W, \Phi) \in \mathrm{FM}(X)} U(W, \Phi) \subset \operatorname{Stab}_{\mathrm{n}}^{\circ}(X / Y),
$$

such that two chambers are either equal or disjoint. Moreover we have $\overline{\mathcal{M}}=$ $\operatorname{Stab}_{\mathrm{n}}^{\circ}(X / Y)$.

Proof. By the definition, $(W, \Phi) \in \mathrm{FM}(X)$ is obtained as a successive sequence of tensoring line bundles and standard equivalences. Therefore Proposition 4.10 implies that the $U(W, \Phi)$ are contained in the same connected component, i.e.

$$
\mathcal{M} \subset \operatorname{Stab}_{\mathrm{n}}^{\circ}(X / Y)
$$

Also by Proposition 4.10 (i), $U(W, \Phi) \cap U\left(W^{\prime}, \Phi^{\prime}\right) \neq \emptyset$ implies $U(W, \Phi)=U\left(W^{\prime}, \Phi^{\prime}\right)$. It remains to show $\overline{\mathcal{M}}=\operatorname{Stab}_{\mathrm{n}}^{\circ}(X / Y)$. Take $\sigma \in \operatorname{Stab}_{\mathrm{n}}^{\circ}(X / Y)$ and $\sigma_{0} \in U_{X}$. Let

$$
\gamma:[0,1] \longrightarrow \operatorname{Stab}_{\mathrm{n}}^{\circ}(X / Y)
$$

be a path such that $\gamma(0)=\sigma_{0}$ and $\gamma(1)=\sigma$. Note that by Theorem 3.3. the chambers in $N^{1}(X / Y)_{\mathbb{C}}$ given by $\phi_{*} A(W / Y)_{\mathbb{C}}$ for crepant small resolutions $g: W \rightarrow$ 
$Y$ are finite. Because the map $\mathcal{Z}_{X}$ is a local homeomorphism by Theorem 2.4 we may assume $\mathcal{Z}_{X}(\gamma)$ passes through only codimension one walls of these chambers by deforming $\gamma$ if necessary. Then there exists a sequence,

$$
0<t_{1}<t_{2}<\cdots<t_{n-1}<1
$$

such that $\mathcal{Z}_{X}\left(\gamma\left(t_{k}\right)\right)$ is contained in one of the codimension one walls, and the $\mathcal{Z}_{X}\left(\gamma\left(\left(t_{k-1}, t_{k}\right)\right)\right)$ are contained in the chambers. Then $\gamma\left(\left(0, t_{1}\right)\right)$ is contained in $U_{X}$ and by Proposition 4.10 (ii), $\gamma\left(\left(t_{1}, t_{2}\right)\right)$ is contained in some $U(W, \Phi)$ with $(W, \Phi) \in \operatorname{FM}(X)$. Repeating this argument, we have $\gamma(t) \in \overline{\mathcal{M}}$ for all $t \in[0,1]$. In particular, $\sigma=\gamma(1) \in \overline{\mathcal{M}}$.

\section{Stability COnditions And COVERING SPACES}

In this section, we give the proof of Theorem 1.2 ,

5.1. Root lattices and Weyl reflections. First we introduce the root lattice associated to the exceptional locus of $f: X \rightarrow Y$.

Definition 5.1. We define the $\mathbb{Z}$-module $\Lambda_{f}$ by

$$
\Lambda_{f}:=\bigoplus_{i=1}^{N} \mathbb{Z} e_{i}
$$

and introduce the pairing $():, \Lambda_{f} \times \Lambda_{f} \rightarrow \mathbb{Z}$ by $\left(e_{i}, e_{i}\right)=-2,\left(e_{i}, e_{j}\right)=1$ if $i \neq j$ and $C_{i} \cap C_{j} \neq \emptyset$, and $\left(e_{i}, e_{j}\right)=0$ if otherwise.

It is well known that the pairing $($,$) on \Lambda_{f}$ is negative definite. We define the sets $V\left(\Lambda_{f}\right), S\left(\Lambda_{f}\right)$ by

$$
V\left(\Lambda_{f}\right):=\left\{v \in \Lambda_{f} \mid(v, v)=-2\right\}, \quad S\left(\Lambda_{f}\right)=\left\{e_{i} \mid 1 \leq i \leq N\right\} .
$$

An element $v \in V\left(\Lambda_{f}\right)$ is called a root, and $e_{i} \in S\left(\Lambda_{f}\right)$ is called a simple root. It is well known that any element $v \in V\left(\Lambda_{f}\right)$ is written as

$$
v=\sum_{i=1}^{N} r_{i} e_{i},
$$

with $r_{i} \in \mathbb{Z}_{\geq 0}$ for all $i$, or $r_{i} \in \mathbb{Z}_{\leq 0}$ for all $i$. For $v \in V\left(\Lambda_{f}\right)$, we associate the reflection

$$
r_{v}: \Lambda_{f} \ni x \longmapsto x+(v, x) v \in \Lambda_{f} .
$$

It is well known that the set $V\left(\Lambda_{f}\right)$ is obtained by applying reflections $\left\{r_{e_{i}}\right\}_{1 \leq i \leq N}$ to the set $S\left(\Lambda_{f}\right)$. Note that we have a natural identification of vector spaces,

$$
\Lambda_{f} \otimes_{\mathbb{Z}} \mathbb{R} \cong N_{1}(X / Y)
$$

by identifying $e_{i}$ with $C_{i}$. Then the dual of $r_{v}$ gives the reflection,

$$
r_{v}^{*}: N^{1}(X / Y) \longrightarrow N^{1}(X / Y),
$$

and is called the Weyl reflection. Let us consider a partial resolution $X \stackrel{f^{\prime}}{\rightarrow} Y^{\prime} \rightarrow Y$. Then there is a natural inclusion $\Lambda_{f^{\prime}} \hookrightarrow \Lambda_{f}$, and it is obvious that

$$
V\left(\Lambda_{f^{\prime}}\right)=V\left(\Lambda_{f}\right) \cap \Lambda_{f^{\prime}} .
$$

Let $g: W \rightarrow Y$ be another crepant small resolution. Then the dual graphs of the exceptional loci of $f$ and $g$ are identified, since they are identified under simple flops. (In our case we flop at single rational curves since $Y$ is complete; thus this 
statement is obvious. However we note that the explicit constructions of flops 24, Theorem 2.4] would also imply this without the assumption that $Y$ is complete.) Under the assumption that $X_{0}$ is smooth, it is possible to choose the identification of the dual graphs canonically (does not depend on a choice of a decomposition of $W \rightarrow X$ into flops) because the birational map $W \rightarrow X$ induces an isomorphism between minimal resolutions, $g^{-1}\left(Y_{0}\right) \stackrel{\cong}{\rightrightarrows} X_{0}$. Hence we have an identification, $\psi: \Lambda_{g} \cong \Lambda_{f}$, which preserves the set of simple roots. By taking its dual, we obtain an isomorphism

$$
\psi^{*}: N^{1}(X / Y) \longrightarrow N^{1}(W / Y) .
$$

By composing with the strict transform, we get the isomorphism

$$
\phi_{*} \circ \psi^{*}: N^{1}(X / Y) \longrightarrow N^{1}(W / Y) \longrightarrow N^{1}(X / Y) \text {. }
$$

If $\phi: W \rightarrow X$ is a flop at $C_{i}$, the above map is calculated in [28, Theorem 6.3], and it coincides with $r_{e_{i}}^{*}$. Here we note that in [28, Theorem 6.3], the assumption that a smooth surface $C \subset X_{0}$ with $(C)_{X_{0}}^{2}=-2$ exists is needed. Combined with Lemma 4.8, we obtain the following.

Lemma 5.2. We have the map

$$
\Theta: \operatorname{FM}(X) \ni u \longmapsto \phi^{u} \circ \psi^{*} \in \operatorname{Aff}\left(N^{1}(X / Y)_{\mathbb{C}}\right) .
$$

Here the right hand side is the group of affine transformations on $N^{1}(X / Y)_{\mathbb{C}}$. Its image coincides with the subgroup generated by Weyl reflections with respect to simple roots of $\Lambda_{f}$ and translations by the lattice $\operatorname{Pic}(X) \subset N^{1}(X / Y)$.

5.2. Proof of Theorem 1.2. Before giving the proof of Theorem 1.2, we introduce some notation. First note that the following set,

$$
G:=\{(W, \Phi) \in \operatorname{FM}(X) \mid W=X\},
$$

is a subgroup of the group of autoequivalences of $D^{b}(X)$. Then the following map,

$$
G \ni(X, \Phi) \longmapsto \operatorname{ch}_{1} \Phi\left(\mathcal{O}_{X}\right) \in N^{1}(X / Y),
$$

is a group homomorphism, by the same argument as in the proof of Lemma 3.15. We define $G^{\circ} \subset G$ to be the kernel of the above map. Next for $v \in V\left(\Lambda_{f}\right)$, define $H_{v} \subset N^{1}(X / Y)_{\mathbb{C}}$ to be

$$
H_{v}:=\left\{\beta+i \omega \in N^{1}(X / Y)_{\mathbb{C}} \mid(\beta+i \omega) v \in \mathbb{Z}\right\} .
$$

Now we prove Theorem 1.2 ,

Theorem 5.3. We have the following map:

$$
\mathcal{Z}_{X}: \operatorname{Stab}_{\mathrm{n}}^{\circ}(X / Y) \longrightarrow N^{1}(X / Y)_{\mathbb{C}} \backslash \bigcup_{v \in V\left(\Lambda_{f}\right)} H_{v} .
$$

It is a regular covering map with Galois group equal to $G^{\circ}$.

Proof. We divide the proof into 3 steps.

Step 1. We have $\operatorname{Im}\left(\mathcal{Z}_{X}\right) \subset N^{1}(X / Y)_{\mathbb{C}} \backslash \bigcup_{v \in V\left(\Lambda_{f}\right)} H_{v}$.

Proof. Note that for $u=(W, \Phi) \in \operatorname{FM}(X)$ with $g: W \rightarrow Y$, $\phi^{u}$ restricts to an isomorphism,

$$
\phi^{u}: N^{1}(W / Y)_{\mathbb{C}} \backslash \bigcup_{v \in V\left(\Lambda_{g}\right)} H_{v} \longrightarrow N^{1}(X / Y)_{\mathbb{C}} \backslash \bigcup_{v \in V\left(\Lambda_{f}\right)} H_{v} .
$$


Therefore by Theorem 4.11 and Lemma 4.8, it suffices to show that for $\sigma=$ $\left(Z_{(\beta, \omega)}, \mathcal{P}\right) \in \bar{U}_{X}$, we have $(\beta+i \omega) v \notin \mathbb{Z}$ for all $v \in V\left(\Lambda_{f}\right)$. First from Proposition 4.4 we have $(\beta+i \omega) v \notin \mathbb{Z}$ for simple roots $v$. Next suppose $\omega$ is written as $\omega=f^{\prime} * \omega^{\prime}$ for a partial resolution $X \stackrel{f^{\prime}}{\rightarrow} Y^{\prime} \rightarrow Y$, and $\omega^{\prime} \in A\left(Y^{\prime} / Y\right)$. Then $(\beta+i \omega) v$ could be an integer only if

$$
v \in V\left(\Lambda_{f}\right) \cap \Lambda_{f^{\prime}}=V\left(\Lambda_{f^{\prime}}\right)
$$

Take $v \in V\left(\Lambda_{f^{\prime}}\right)$. Then by the argument of Lemma [5.2, there exist flops over $Y^{\prime}$,

$$
\phi: W=X^{n} \rightarrow X^{n-1} \rightarrow \cdots \rightarrow X^{0}=X,
$$

and a simple root $v^{\prime} \in S\left(\Lambda_{g^{\prime}}\right)$ such that $\phi_{*} H_{v^{\prime}}=H_{v}$. Here $g^{\prime}$ is a structure morphism $g^{\prime}: W \rightarrow Y^{\prime}$. Note that

$$
\omega \in f^{\prime *} A\left(Y^{\prime} / Y\right) \subset \phi_{*} \bar{A}(W / Y) .
$$

Using Theorem 4.11 and the fact that $\mathcal{Z}_{X}$ is a local isomorphism, we can find a region $U(W, \Phi)$ such that $\sigma \in \bar{U}(W, \Phi)$. Since $\mathcal{Z}_{W}\left(\bar{U}_{W}\right) \subset N^{1}(W / Y)_{\mathbb{C}} \backslash H_{v^{\prime}}$, Lemma 4.8 implies

$$
\beta+i \omega=\mathcal{Z}_{X}(\sigma) \in \mathcal{Z}_{X}(\bar{U}(W, \Phi)) \subset N^{1}(X / Y)_{\mathbb{C}} \backslash H_{v}
$$

This implies $(\beta+i \omega) v \notin \mathbb{Z}$.

Step 2. The map $\mathcal{Z}_{X}$ is surjective.

Proof. By Lemma 4.8, it suffices to show surjectivity on $\bar{A}(X / Y)_{\mathbb{C}} \backslash \bigcup_{v \in V\left(\Lambda_{f}\right)} H_{v}$. Let us take $\beta+i \omega \in \bar{A}(X / Y)_{\mathbb{C}}$ and suppose $\omega=f^{\prime}{ }^{*} \omega^{\prime}$ for a partial resolution $X \stackrel{f^{\prime}}{\rightarrow} Y^{\prime} \rightarrow Y$; i.e. $\beta+i \omega$ lies in the wall $\mathcal{W}:=N^{1}(X / Y) \oplus i f^{\prime}{ }^{*} A\left(Y^{\prime} / Y\right)$. For a root $v \in V\left(\Lambda_{f^{\prime}}\right)$, let $\bar{H}_{v}$ be

$$
\bar{H}_{v}:=\left\{\beta \in N^{1}\left(X / Y^{\prime}\right) \mid \beta \cdot v \in \mathbb{Z}\right\} \subset N^{1}\left(X / Y^{\prime}\right) .
$$

$\bar{H}_{v}$ is a real codimension one hypersurface and we have the fiber space structure

$$
\Pi: \mathcal{W} \backslash \bigcup_{v \in V\left(\Lambda_{f}\right)} H_{v} \longrightarrow N^{1}\left(X / Y^{\prime}\right) \backslash \bigcup_{v \in V\left(\Lambda_{f^{\prime}}\right)} \bar{H}_{v},
$$

with fiber $f^{\prime *} A\left(Y^{\prime} / Y\right)_{\mathbb{C}}$. Let $C\left(X / Y^{\prime}\right)$ be one of the connected components of the right hand side that contains the following set:

$$
\left\{\beta \in N^{1}\left(X / Y^{\prime}\right) \mid-\varepsilon<\beta \cdot v<0 \text { for all } v \in S\left(\Lambda_{f^{\prime}}\right)\right\},
$$

for sufficiently small $0<\varepsilon \ll 1$. We are now going to argue that it suffices to check the surjectivity on $\Pi^{-1}\left(C\left(X / Y^{\prime}\right)\right)$.

Let $\mathcal{G}$ be the group of Weyl reflections generated by the reflections by the simple roots $S\left(\Lambda_{f^{\prime}}\right)$, acting on $N^{1}\left(X / Y^{\prime}\right)$. Let $\operatorname{Pic}\left(X / Y^{\prime}\right) \subset N^{1}\left(X / Y^{\prime}\right)$ be the lattice consisting of numerical classes of line bundles. Then the action of $\mathcal{G}$ preserves $\operatorname{Pic}\left(X / Y^{\prime}\right)$. Hence we can consider the semi-direct product $\mathcal{G} \ltimes \operatorname{Pic}\left(X / Y^{\prime}\right)$ with its action on $N^{1}\left(X / Y^{\prime}\right) \backslash \bigcup_{v \in V\left(\Lambda_{f^{\prime}}\right)} \bar{H}_{v}$ given by the following formula:

$$
(g, \mathcal{L}) * D=g(D)+\mathcal{L} .
$$

Here $g \in \mathcal{G}, \mathcal{L} \in \operatorname{Pic}\left(X / Y^{\prime}\right)$ and $D \in N^{1}\left(X / Y^{\prime}\right)$. This action also induces the action on the set of connected components of $N^{1}\left(X / Y^{\prime}\right) \backslash \bigcup_{v \in V\left(\Lambda_{f^{\prime}}\right)} \bar{H}_{v}$, which can be easily checked to be transitive. 
On the other hand, let us use Lemma[5.2. Then for each $(\phi, \mathcal{L}) \in \mathcal{G} \ltimes \operatorname{Pic}\left(X / Y^{\prime}\right)$, we can find $(W, \Phi) \in \operatorname{FM}(X)$ such that

- A successive sequence

$$
W=X^{n} \rightarrow X^{n-1} \rightarrow \cdots \rightarrow X^{1} \rightarrow X^{0}=X,
$$

of Definition 3.13 is defined over $Y^{\prime}$, i.e. the structure morphism $X^{j} \rightarrow Y$ factors as $X^{j} \rightarrow Y^{\prime} \rightarrow Y$. In particular there exists a morphism $g^{\prime}: W \rightarrow$ $Y^{\prime}$ and $\Theta(W, \Phi)$ preserves the wall $\mathcal{W}$.

- We have $\left.\Pi \circ \Theta(W, \Phi)\right|_{\mathcal{W}}=(\phi, \mathcal{L}) \circ \Pi$.

Also note that for any lattice isomorphism $\psi: \Lambda_{g^{\prime}} \rightarrow \Lambda_{f^{\prime}}$ which preserves the simple roots, the induced morphism $\psi^{*}: N^{1}\left(X / Y^{\prime}\right) \rightarrow N^{1}\left(W / Y^{\prime}\right)$ takes $C\left(X / Y^{\prime}\right)$ to $C\left(W / Y^{\prime}\right)$. Therefore using Lemma 4.8, we may assume $\Pi(\beta+i \omega)$ lies in $C\left(X / Y^{\prime}\right)$. In this region, let us take the pair:

$$
\sigma:=\left(Z_{(\beta, \omega)},{ }^{0} \operatorname{Per}\left(X / Y^{\prime}\right) \cap \mathcal{D}_{X / Y}\right) .
$$

By the same proof as in Lemma 4.3 and Proposition 4.4, we can conclude $\sigma \in$ $\operatorname{Stab}_{\mathrm{n}}^{\circ}(X / Y)$.

Step 3. The map $\mathcal{Z}_{X}$ is a regular covering map with Galois group $G^{\circ}$.

Proof. First for $\sigma \in U_{X}$ and $u=(X, \Phi) \in G^{\circ}$, one has

$$
\Phi_{*} u \in U(X, \Phi) \subset \operatorname{Stab}_{\mathrm{n}}^{\circ}(X / Y) .
$$

Therefore the action of $G^{\circ}$ on the set of stability conditions preserves the connected component $\operatorname{Stab}_{\mathrm{n}}^{\circ}(X / Y)$. Moreover by the definition of $G^{\circ}$, one has $\phi^{u}=$ id. Therefore Lemma 4.8 implies that $G^{\circ}$ acts on $\operatorname{Stab}_{\mathrm{n}}^{\circ}(X / Y)$ as deck transformations. This action is also faithful. In fact, assume that for $(X, \Phi) \in G^{\circ}$, one has $\Phi_{*} \sigma=\sigma$ for some $\sigma \in \operatorname{Stab}_{\mathrm{n}}^{\circ}(X / Y)$. By Theorem 4.11 we may assume $\sigma \in U\left(W, \Phi^{\prime}\right)$ for some $\left(W, \Phi^{\prime}\right) \in \operatorname{FM}(X)$. Then

$$
U\left(W, \Phi^{\prime}\right) \cap U\left(W, \Phi \circ \Phi^{\prime}\right) \neq \emptyset .
$$

By Proposition 4.10 (i), $\Phi$ is given by tensoring the line bundle. Because $\operatorname{ch}_{1} \Phi\left(\mathcal{O}_{X}\right)$ $=0$, we have $\Phi=$ id.

Next let us take $\sigma, \sigma^{\prime} \in \operatorname{Stab}_{\mathrm{n}}^{\circ}(X / Y)$ such that $\mathcal{Z}_{X}(\sigma)=\mathcal{Z}_{X}\left(\sigma^{\prime}\right)$. By Theorem 4.11, there exist some regions $U(W, \Phi), U\left(W, \Phi^{\prime}\right)$ such that $\sigma \in \bar{U}(W, \Phi)$ and $\sigma^{\prime} \in \bar{U}\left(W, \Phi^{\prime}\right)$. Let us choose a sequence $\left\{\sigma_{k}\right\}_{k=1}^{\infty} \subset U(W, \Phi)$ which converges to $\sigma$. Then there exists another sequence $\left\{\sigma_{k}^{\prime}\right\}_{k=1}^{\infty} \subset U\left(W, \Phi^{\prime}\right)$ which converges to $\sigma^{\prime}$ and $\mathcal{Z}_{X}\left(\sigma_{k}\right)=\mathcal{Z}_{X}\left(\sigma_{k}^{\prime}\right)$. Let us take $\mathcal{L} \in \operatorname{Pic}(W)$ to be

$$
\mathcal{L}=\operatorname{det} \Phi^{\prime-1}\left(\mathcal{O}_{X}\right) \otimes\left\{\operatorname{det} \Phi^{-1}\left(\mathcal{O}_{X}\right)\right\}^{-1} .
$$

Then by Lemma 6.1 below, the functor

$$
\Phi^{\prime \prime}:=\Phi^{\prime} \circ \otimes \mathcal{L} \circ \Phi^{-1}: D^{b}(X) \rightarrow D^{b}(X)
$$

satisfies $\left.\Phi^{\prime \prime}\left(\mathcal{O}_{X}\right)\right|_{X^{\circ}} \cong \mathcal{O}_{X^{\circ}}$, where $X^{\circ}=X \backslash C$. Because $C \subset X$ is codimension two, this implies $\operatorname{det} \Phi^{\prime \prime}\left(\mathcal{O}_{X}\right) \cong \mathcal{O}_{X}$; hence $\operatorname{ch}_{1} \Phi^{\prime \prime}\left(\mathcal{O}_{X}\right)=0$. Therefore $\left(X, \Phi^{\prime \prime}\right) \in$ $G^{\circ}$ and

$$
\mathcal{Z}_{X}\left(\Phi_{*}^{\prime \prime}\left(\sigma_{k}\right)\right)=\mathcal{Z}_{X}\left(\sigma_{k}\right)=\mathcal{Z}_{X}\left(\sigma_{k}^{\prime}\right) .
$$

Note that $\Phi_{*}^{\prime \prime}$ takes $U(W, \Phi)$ to $U\left(W, \Phi^{\prime}\right)$; thus $\Phi_{*}^{\prime \prime}\left(\sigma_{k}\right)$ is contained in $U\left(W, \Phi^{\prime}\right)$. Since $\mathcal{Z}_{X}$ is injective on $U\left(W, \Phi^{\prime}\right)$, it follows that

$$
\Phi_{*}^{\prime \prime}\left(\sigma_{k}\right)=\sigma_{k}^{\prime} .
$$


Taking the limit $k \rightarrow \infty$, we get $\Phi_{*}^{\prime \prime}(\sigma)=\sigma^{\prime}$. This shows $\mathcal{Z}_{X}$ is a regular covering map with Galois group $G^{\circ}$.

Theorem 5.3 provides some information on the group of autoequivalences of $\mathcal{D}_{X / Y}$. Let $\operatorname{Aut}^{\circ}\left(\mathcal{D}_{X / Y}\right)$ be the group of autoequivalences $\Phi$ of $\mathcal{D}_{X / Y}$ such that

- $\Phi$ preserves the class $\left[\mathcal{O}_{x}\right] \in K\left(\mathcal{D}_{X / Y}\right)$;

- $\Phi$ preserves the connected component $\operatorname{Stab}_{\mathrm{n}}^{\circ}(X / Y)$.

Then we have the following.

Corollary 5.4. (i) We have the group isomorphism

$$
G=\left\{(W, \Phi) \in \mathrm{FM}^{\circ}(X) \mid W=X\right\} \cong \operatorname{Aut}^{\circ}\left(\mathcal{D}_{X / Y}\right) .
$$

(ii) There exists the following exact sequence:

$$
\pi_{1}\left(N^{1}(X / Y)_{\mathbb{C}} \backslash \bigcup_{v \in V\left(\Lambda_{f}\right)} H_{v}\right) \longrightarrow \operatorname{Aut}^{\circ}\left(\mathcal{D}_{X / Y}\right) \longrightarrow \operatorname{Pic}(X) \longrightarrow 0 .
$$

Here the last map is defined by taking $\Phi$ to $\operatorname{det} \Phi\left(\mathcal{O}_{X}\right)$.

Proof. (i) Let us take $\Phi \in \operatorname{Aut}^{\circ}\left(\mathcal{D}_{X / Y}\right)$ and $\sigma \in U_{X}$. Then $\Phi_{*} \sigma \in \operatorname{Stab}_{\mathrm{n}}^{\circ}(X / Y)$. By Theorem 4.11, $\Phi_{*} \sigma \in \bar{U}\left(W, \Phi^{\prime}\right)$ for some $\left(W, \Phi^{\prime}\right) \in \mathrm{FM}(X)$. By deforming $\sigma$ if necessary, we may assume $\Phi_{*} \sigma \in U\left(W, \Phi^{\prime}\right)$. Then we have

$$
\Phi_{*} \sigma \in U(X, \Phi) \cap U\left(W, \Phi^{\prime}\right) \neq \emptyset .
$$

Thus by Proposition 4.10(i), we have $W \cong X$ and $\Phi \cong \otimes \mathcal{L} \circ \Phi^{\prime}$ for some $\mathcal{L} \in \operatorname{Pic}(X)$. Therefore $(X, \Phi) \in \operatorname{FM}(X)$.

(ii) This follows from (i) and Theorem 5.3 .

Remark 5.5. If we knew $\operatorname{Stab}_{\mathrm{n}}^{\circ}(X / Y)$ to be simply connected, we would have the exact sequence:

$$
0 \longrightarrow \pi_{1}\left(N^{1}(X / Y)_{\mathbb{C}} \backslash \bigcup_{v \in V\left(\Lambda_{f}\right)} H_{v}\right) \longrightarrow \operatorname{Aut}^{\circ}\left(\mathcal{D}_{X / Y}\right) \longrightarrow \operatorname{Pic}(X) \longrightarrow 0 .
$$

Example. Let $Y=\operatorname{Spec} \mathbb{C}[[x, y, z, w]] /(x y-z w)$ and $f: X \rightarrow Y$ be the blowing up at the ideal $(x, z)$ as in the introduction. Then Theorem 5.3 describes $\operatorname{Stab}_{\mathrm{n}}^{\circ}(X / Y)$ as a covering map,

$$
\operatorname{Stab}_{\mathrm{n}}^{\circ}(X / Y) \longrightarrow \mathbb{C} \backslash \mathbb{Z} .
$$

In this case, $\operatorname{Pic}(X)=\mathbb{Z}$, and the action of $n \in \operatorname{Pic}(X)$ on $N^{1}(X / Y)_{\mathbb{C}}=\mathbb{C}$ is given by the translation, $\beta+i \omega \mapsto n+\beta+i \omega$. Therefore Corollary 5.4 implies

$$
\begin{aligned}
\operatorname{Stab}_{\mathrm{n}}^{\circ}(X / Y) / \operatorname{Aut}^{\circ}\left(\mathcal{D}_{X / Y}\right) & \cong(\mathbb{C} \backslash \mathbb{Z}) / \mathbb{Z} \\
& \cong \mathbb{P}^{1} \backslash\{\text { three points }\} .
\end{aligned}
$$

Thus we have obtained the same picture as in [1, Figure 2].

Remark 5.6. If we don't assume the existence of a smooth surface $X_{0}$, the description may change. For example, there is an example of a crepant small resolution $f: X \rightarrow Y$ which contracts a $(1,-3)$-curve and its scheme theoretic fiber is nonreduced [28, Lemma 5.16]. In this case we have to delete extra rational points from $\mathbb{C} \backslash \mathbb{Z}$, according to the proof of Lemma 4.3 . Thus Theorem 5.3 is false in this case. At this time, the author does not know how to describe $\operatorname{Stab}_{\mathrm{n}}^{\circ}(X / Y)$ in a beautiful way in a general situation involving such cases. 


\section{Some technicAl LEMmas}

In this section, we give the postponed proofs.

\subsection{Proof of Lemma 3.8 .}

Proof. (i) For simplicity we show the case of $p=0$. The objects of ${ }^{0} \operatorname{Per}\left(\mathcal{D}_{X / Y_{i}}\right)$ are contained in the right hand side by the definition. Take $E \in \operatorname{Coh}_{\cup_{j \neq i} C_{j}}(X)$. Then $\mathbf{R} g_{i *} E \in D^{b}\left(Y_{i}\right)$ is a sheaf; hence Lemma 3.5 applied to $g_{i}: X \rightarrow Y_{i}$ implies $E \in{ }^{0} \operatorname{Per}\left(X / Y_{i}\right)$. Since $E$ is also supported on $C, E$ is contained in the right hand side.

Conversely let $\mathcal{B} \subset \mathcal{D}_{X / Y}$ be the smallest extension closed subcategory which contains the left hand side. Let us take $A \in{ }^{0} \operatorname{Per}\left(X / Y_{i}\right) \cap \mathcal{D}_{X / Y}$. Then by Lemma 3.5(i), we have the distinguished triangle,

$$
H^{-1}(A)[1] \longrightarrow A \longrightarrow H^{0}(A) \longrightarrow H^{-1}(A)[2] .
$$

The conditions of Lemma 3.5 applied to $g_{i}: X \rightarrow Y_{i}$ show that $H^{-1}(A)[1]$ is also contained in ${ }^{0} \operatorname{Per}\left(X / Y_{i}\right)$. By Lemma 3.5(ii), one has $g_{i *} H^{-1}(A)=0$. Therefore $H^{-1}(A)$ must be supported on $C_{i}$; hence

$$
H^{-1}(A)[1] \in{ }^{0} \operatorname{Per}\left(\mathcal{D}_{X / Y_{i}}\right) \subset \mathcal{B} .
$$

Therefore it is enough to check $H^{0}(A) \in \mathcal{B}$. Let $I_{C} \subset I_{C_{i}} \subset \mathcal{O}_{X}$ be the ideal sheaves which define $C, C_{i}$ respectively. Since $H^{0}(A)$ is supported on $C$, it is annihilated by $I_{C}^{n}$ for some $n \in \mathbb{N}$. We have the exact sequence,

$$
0 \rightarrow I_{C_{i}}^{n} / I_{C}^{n} \longrightarrow \mathcal{O}_{X} / I_{C}^{n} \longrightarrow \mathcal{O}_{X} / I_{C_{i}}^{n} \longrightarrow 0 .
$$

Note that $I_{C_{i}}^{n} / I_{C}^{n}$ is supported on $\bigcup_{j \neq i} C_{j}$ and $\mathcal{O}_{X} / I_{C_{i}}^{n}$ is supported on $C_{i}$. By tensoring $H^{0}(A)$, we get the exact sequence

$$
0 \longrightarrow A_{1} \longrightarrow H^{0}(A) \longrightarrow A_{2} \longrightarrow 0
$$

where $A_{2}=H^{0}(A) \otimes \mathcal{O}_{X} / I_{C_{i}}^{n}$ and $A_{1}$ is the image of the map $H^{0}(A) \otimes I_{C_{i}}^{n} / I_{C}^{n} \rightarrow$ $H^{0}(A)$. Note that $A_{1} \in \operatorname{Coh}_{\cup_{j \neq i} C_{j}}(X) \subset \mathcal{B}$ and $A_{2} \in \operatorname{Coh}_{C_{i}}(X)$. On the other hand, since $R^{1} g_{i *} H^{0}(A)=0$ by Lemma 3.5(ii), the long exact sequence associated to the above sequence gives $R^{1} g_{i *} A_{2}=0$. Therefore $A_{2}$ satisfies the conditions of Lemma 3.5. thus $A_{2} \in{ }^{0} \operatorname{Per}\left(\mathcal{D}_{X / Y_{i}}\right) \subset \mathcal{B}$. Therefore by the exact sequence above, we have $H^{0}(A) \in \mathcal{B}$.

(ii) Note that the restriction of $g_{i}: X \rightarrow Y_{i}$ to $X_{0}$ is a minimal resolution of $A_{1}$-singularity. Thus the scheme theoretic fiber of $g_{i}$ is reduced. Hence by (i) and Proposition 3.6. the left hand side is the smallest extension closed subcategory of $\mathcal{D}_{X / Y}$ which contains

$$
\operatorname{Coh}_{\cup_{j \neq i} C_{j}}(X) \cup\left\{\mathcal{O}_{C_{i}}, \mathcal{O}_{C_{i}}(-1)[1]\right\} .
$$

Similarly the right hand side is the smallest extension closed subcategory of $\mathcal{D}_{X / Y}$ which contains

$$
\begin{aligned}
& \operatorname{Coh}_{\cup_{j \neq i} C_{j}}(X) \cup\left\{\mathcal{O}_{C_{i}}(-2)[1], \mathcal{O}_{C_{i}}(-1)\right\} \otimes \mathcal{O}_{X}\left(D_{i}\right) \\
& =\operatorname{Coh}_{\cup_{j \neq i} C_{j}}(X) \cup\left\{\mathcal{O}_{C_{i}}(-1)[1], \mathcal{O}_{C_{i}}\right\} .
\end{aligned}
$$

Therefore both sides coincide.

(iii) Since the scheme theoretic fiber of $g_{i}$ is reduced, this follows from (i) and Proposition 3.6 . 


\subsection{Proof of Lemma 3.14.}

Proof. (i) First we check that $\left[\mathcal{O}_{x}\right]$ does not depend on a choice of $x \in C$. Since $\left[\mathcal{O}_{x}\right]=\left[\mathcal{O}_{C_{i}}\right]-\left[\mathcal{O}_{C_{i}}(-1)\right]$ if $x \in C_{i},\left[\mathcal{O}_{x}\right]$ does not depend on $x \in C_{i}$. Moreover since $C$ is connected, one can conclude $\left[\mathcal{O}_{x}\right]$ does not depend on $x \in C$.

Next one has

$$
\chi\left(\mathcal{O}_{X}, \mathcal{O}_{x}\right)=1, \quad \chi\left(\mathcal{O}_{X}, \mathcal{O}_{C_{i}}(-1)\right)=0, \quad \chi\left(\mathcal{O}_{X}\left(D_{j}\right), \mathcal{O}_{C_{i}}(-1)\right)=-\delta^{i j} .
$$

Here $\delta^{i j}=1$ if $i=j$ and zero if $i \neq j$. Therefore $\left\{\left[\mathcal{O}_{x}\right],\left[\mathcal{O}_{C_{i}}(-1)\right]\right\}_{1 \leq i \leq N}$ are linearly independent.

Finally, we check that the set $\left\{\left[\mathcal{O}_{x}\right],\left[\mathcal{O}_{C_{i}}(-1)\right]\right\}_{1 \leq i \leq N}$ spans $K\left(\mathcal{D}_{X / Y}\right)$. For $E \in \mathcal{D}_{X / Y}$, we can write $[E]=\sum(-1)^{i}\left[H^{i}(E)\right]$ in $K\left(\mathcal{D}_{X / Y}\right)$. So we assume $E \in \operatorname{Coh}_{C}(X)$. Then one has a filtration

$$
E_{0} \subset E_{1} \subset \cdots \subset E_{n-1} \subset E_{n}=E
$$

such that $F_{j}=E_{j} / E_{j-1}$ is an $\mathcal{O}_{C_{i}}$-module for some $i$. Since $\left[F_{j}\right]$ is written as a sum of $\left[\mathcal{O}_{x_{i}}\right]$ for $x_{i} \in C_{i}$ and $\left[\mathcal{O}_{C_{i}}(-1)\right],[E]$ is written as a linear combination of $\left\{\left[\mathcal{O}_{x}\right],\left[\mathcal{O}_{C_{i}}(-1)\right]\right\}_{1 \leq i \leq N}$.

(ii) By the definition of $\operatorname{FM}(X), \Phi$ is decomposed into tensoring line bundles and standard equivalences. Cleary tensoring a line bundle preserves the class $\left[\mathcal{O}_{x}\right]$. Also a standard equivalence preserves the class $\left[\mathcal{O}_{x}\right]$, because $\Phi$ takes point sheaves to point sheaves away from a flopped curve.

6.3. Proof of Lemma 3.15. First we introduce some notation and prepare a lemma. For each crepant small resolution $g: W \rightarrow Y$, let $Y^{\circ}:=Y \backslash\{0\}, W^{\circ}:=$ $W \backslash \operatorname{Ex}(g)$ and $j_{W}: W^{\circ} \hookrightarrow W$ be the open immersion. Here $\operatorname{Ex}(g)$ is the exceptional locus of $g$. Let us take $(W, \Phi) \in \operatorname{FM}(X)$. Note that the birational map $\phi: W \rightarrow X$ gives an isomorphism $\phi^{\circ}: W^{\circ} \rightarrow X^{\circ}$. It seems the following lemma is well known, but we give the proof for lack of a reference.

Lemma 6.1. There exists an equivalence $\Phi^{\circ}: D^{b}\left(W^{\circ}\right) \rightarrow D^{b}\left(X^{\circ}\right)$ such that the following diagram commutes:

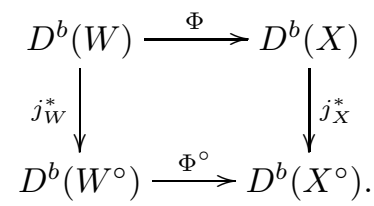

More precisely, $\Phi^{\circ}\left(\mathcal{O}_{W^{\circ}}\right)$ is a line bundle, and for $E \in D^{b}\left(W^{\circ}\right)$ we have

$$
\Phi^{\circ}(E) \cong \phi_{*}^{\circ} E \otimes \Phi^{\circ}\left(\mathcal{O}_{W^{\circ}}\right) .
$$

Proof. Let $\mathcal{E} \in D^{b}(X \times W)$ be the kernel of $\Phi$. Define $\mathcal{E}^{\circ}$ to be

$$
\mathcal{E}^{\circ}:=\left.\mathcal{E}\right|_{X \circ \times W^{\circ}} \in D^{b}\left(X^{\circ} \times W^{\circ}\right) \text {. }
$$

Then let $\Phi^{\circ}$ be

$$
\Phi^{\circ}:=\Phi_{W^{\circ} \rightarrow X^{\circ}}^{\mathcal{E}^{\circ}}: D^{b}\left(W^{\circ}\right) \longrightarrow D^{b}\left(X^{\circ}\right) .
$$

We check that the above diagram commutes. Let $p_{X}^{\circ}, p_{W}^{\circ}$ be the projections from $X^{\circ} \times W^{\circ}$ onto $X^{\circ}$ and $W^{\circ}$, and $p_{X}^{\prime}: X^{\circ} \times W \rightarrow X^{\circ}$ be the projection. For 
$E \in D^{b}(W)$, one has

$$
\begin{aligned}
j_{X}^{*} \Phi(E) & \cong j_{X}^{*} \mathbf{R} p_{X *}\left(p_{W}^{*}(E) \stackrel{\mathbf{L}}{\otimes} \mathcal{E}\right) \\
& \cong \mathbf{R} p_{X *}^{\prime}\left\{\left.\left(p_{W}^{*}(E) \otimes \mathcal{L}\right)\right|_{X^{\circ} \times W}\right\} \\
& \cong \mathbf{R} p_{X *}^{\circ}\left\{\left.\left(p_{W}^{*}(E) \stackrel{\mathbf{L}}{\otimes} \mathcal{E}\right)\right|_{X^{\circ} \times W^{\circ}}\right\} \\
& \cong \mathbf{R} p_{X *}^{\circ}\left\{p_{W}^{\circ *} j_{W}^{*} E \stackrel{\mathbf{L}}{\otimes} \mathcal{E}^{\circ}\right\} \\
& \cong \Phi^{\circ} \circ j_{W}^{*}(E) .
\end{aligned}
$$

Here the second isomorphism follows from the base change formula for the diagram below,

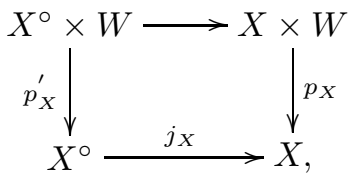

and the third isomorphism follows because $\mathcal{E}$ is supported on $X \times_{Y} W$.

Finally we check that $\Phi^{\circ}$ gives an equivalence. If $\Phi$ is given by tensoring $\mathcal{L} \in$ $\operatorname{Pic}(X)$, then $\Phi^{\circ}$ is also given by tensoring $\left.\mathcal{L}\right|_{X^{\circ}}$. If $\Phi$ is given by the standard equivalence, $\Phi^{\circ}$ is given by $\phi_{*}^{\circ}$. Therefore in these cases, $\Phi^{\circ}$ is an equivalence. Also if we take $\left(W^{\prime}, \Phi^{\prime}\right) \in \operatorname{FM}(W)$, then it is easily checked that

$$
\Phi^{\circ} \circ \Phi^{\prime \circ} \cong\left(\Phi \circ \Phi^{\prime}\right)^{\circ}: D^{b}\left(W^{\prime \circ}\right) \longrightarrow D^{b}\left(W^{\circ}\right) .
$$

Therefore by the definition of $\operatorname{FM}(X)$, the functor $\Phi^{\circ}$ gives an equivalence. Moreover by the description of $\Phi^{\circ}$ for types I and II in Definition 3.13, for any $(W, \Phi) \in$ $\operatorname{FM}(X), \Phi^{\circ}$ is given by

$$
\Phi^{\circ}(E) \cong \phi_{*}^{\circ}(E) \otimes \mathcal{L},
$$

for some $\mathcal{L} \in \operatorname{Pic}\left(X^{\circ}\right)$. Applying $E=\mathcal{O}_{W^{\circ}}$, one has $\mathcal{L}=\Phi^{\circ}\left(\mathcal{O}_{W^{\circ}}\right)$.

\section{Proof of Lemma 3.15}

For $E \in D^{b}(X)$, let $\operatorname{det}(E) \in \operatorname{Pic}(X)$ be the determinant line bundle. Because $\operatorname{ch}_{1} E=\operatorname{ch}_{1} \operatorname{det}(E)$ and $\operatorname{ch}_{0} \Phi\left(\mathcal{O}_{W}\right)=1$, it is sufficient to prove

$$
\operatorname{det} \Phi(\mathcal{L}) \cong \operatorname{det}\left(\phi_{*} \mathcal{L} \otimes \Phi\left(\mathcal{O}_{W}\right)\right) .
$$

Since $C \subset X$ has codimension two, one has $j_{X *} j_{X}^{*} E \cong E$ for a vector bundle $E$ on $X$. Hence it is enough to show the above formula on $X^{\circ}$. On the other hand, by Lemma 6.1 we have

$$
\begin{aligned}
\left.\Phi(\mathcal{L})\right|_{X^{\circ}} & \cong \Phi^{\circ}\left(\left.\mathcal{L}\right|_{W^{\circ}}\right) \\
& \cong \phi_{*}^{\circ}\left(\left.\mathcal{L}\right|_{W^{\circ}}\right) \otimes \Phi^{\circ}\left(\mathcal{O}_{W^{\circ}}\right) \\
& \left.\cong\left(\phi_{*} \mathcal{L} \otimes \Phi\left(\mathcal{O}_{W}\right)\right)\right|_{X^{\circ}} .
\end{aligned}
$$

Note that taking determinants and the restrictions to $X^{\circ}$ commute. Thus one has

$$
\begin{aligned}
\left.\operatorname{det}(\Phi(\mathcal{L}))\right|_{X^{\circ}} & \cong \operatorname{det}\left(\left.\Phi(\mathcal{L})\right|_{X^{\circ}}\right) \\
& \cong \operatorname{det}\left(\left.\left(\phi_{*} \mathcal{L} \otimes \Phi\left(\mathcal{O}_{W}\right)\right)\right|_{X^{\circ}}\right) \\
& \left.\cong \operatorname{det}\left(\phi_{*} \mathcal{L} \otimes \Phi\left(\mathcal{O}_{W}\right)\right)\right|_{X^{\circ}},
\end{aligned}
$$

and the lemma follows. 
6.4. Proof of Lemma 4.5. Before proving Lemma 4.5 we prepare some lemmas. First we give the following technical lemma on the perverse t-structures. Recall that by the definition, $\mathcal{A}_{(i, 0)}$ is given by

$$
\mathcal{A}_{(i, 0)}={ }^{0} \operatorname{Per}\left(X / Y_{i}\right) \cap \mathcal{D}_{X / Y} .
$$

Lemma 6.2. (i) For any object $E \in \mathcal{A}_{(i, 0)}$, the following sequence is an exact sequence in $\mathcal{A}_{(i, 0)}$ :

$$
0 \longrightarrow H^{-1}(E)[1] \longrightarrow E \longrightarrow H^{0}(E) \longrightarrow 0
$$

with $H^{-1}(E)[1] \in{ }^{0} \operatorname{Per}\left(\mathcal{D}_{X / Y_{i}}\right)$.

(ii) For any object $E \in \mathrm{Coh}_{C}(X)$, there exists an exact sequence

$$
0 \longrightarrow F \longrightarrow E \longrightarrow G \longrightarrow 0,
$$

in $\operatorname{Coh}_{C}(X)$ such that

$$
F \in \mathcal{A}_{(i, 0)} \cap \operatorname{Coh}_{C}(X), \quad G[1] \in{ }^{0} \operatorname{Per}\left(\mathcal{D}_{X / Y_{i}}\right) .
$$

Proof. (i) By the criterion of Lemma 3.5 applied to $g_{i}: X \rightarrow Y_{i}$, for $E \in \mathcal{A}_{(i, 0)}$ the objects

$$
H^{-1}(E)[1], \quad H^{0}(E)
$$

are also contained in $\mathcal{A}_{(i, 0)}$. Because $\mathcal{A}_{(i, 0)}$ is a heart of a bounded t-structure on $\mathcal{D}_{X / Y}$, the distinguished triangle

$$
H^{-1}(E)[1] \longrightarrow E \longrightarrow H^{0}(E)
$$

gives a short exact sequence in $\mathcal{A}_{(i, 0)}$. By Lemma 3.5 (ii), we have $g_{i *} H^{-1}(E)=0$. Therefore $H^{-1}(E)$ is supported on $C_{i}$.

(ii) For this proof, we refer to [12, Section 3]. In fact, according to [12, Section 3], the category ${ }^{0} \operatorname{Per}\left(X / Y_{i}\right)$ is obtained from $\operatorname{Coh}(X)$ as a tilting [16] for a certain torsion pair $\left(\mathcal{T}_{0}, \mathcal{F}_{0}\right)$ on $\operatorname{Coh}(X)$, with $\mathcal{T}_{0}$ a torsion part and $\mathcal{F}_{0}$ a free part. According to [12, Section 3], an object $F \in \mathcal{F}_{0}$ must satisfy $g_{i *} F=0$. Therefore $F$ is supported on $C_{i}$, and this shows the torsion theory that $\left(\mathcal{T}_{0}, \mathcal{F}_{0}\right)$ induces the torsion pair $\left(\mathcal{T}_{0}^{\prime}, \mathcal{F}_{0}^{\prime}\right)$ on $\operatorname{Coh}_{C}(X)$. Clearly, the corresponding tilting is $\mathcal{A}_{(i, 0)}$. Therefore we can decompose $E \in \operatorname{Coh}_{C}(X)$ into the exact sequence

$$
0 \longrightarrow T \longrightarrow E \longrightarrow F \longrightarrow 0
$$

such that $T \in \mathcal{T}_{0}^{\prime}$ and $F \in \mathcal{F}_{0}^{\prime}$. By the construction of the tilting [16, Proposition 2.1], $T$ and $F$ [1] are objects in $\mathcal{A}_{(i, 0)}$. Because $F$ must be supported on $C_{i}$, one has $F[1] \in{ }^{0} \operatorname{Per}\left(\mathcal{D}_{X / Y_{i}}\right)$.

We use the assumption that $X_{0}$ is smooth in the following lemma.

Lemma 6.3. There exists a constant $K>0$ such that if $E \in \operatorname{Coh}_{C}(X)$ satisfies $\operatorname{Hom}(E, E)=\mathbb{C}$, we have

$$
\operatorname{ch}_{2}(E)=\sum_{i=1}^{N} r_{i} C_{i}
$$

with $0 \leq r_{i} \leq K$. 


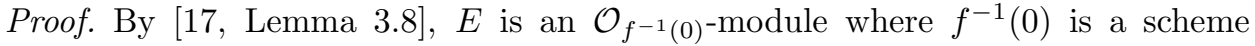
theoretic fiber of $f$ at the closed point $0 \in Y$. In particular for a hyperplane section $0 \in Y_{0} \subset Y$ and its pull back $X_{0}=f^{-1}\left(Y_{0}\right), E$ is an $\mathcal{O}_{X_{0}}$-module. Let us regard $E$ as an object of $\operatorname{Coh}\left(X_{0}\right)$. Note that we have

$$
\operatorname{Hom}_{X}(E, E)=\operatorname{Hom}_{X_{0}}(E, E)=\mathbb{C} .
$$

Because we assume $X_{0}$ is smooth, we can apply the Riemann-Roch theorem on $X_{0}$, and we get

$$
\begin{aligned}
-\operatorname{ch}_{1}(E)^{2} & =\sum(-1)^{i} \operatorname{dim} \operatorname{Ext}_{X_{0}}^{i}(E, E) \\
& \leq 2 .
\end{aligned}
$$

Here we have taken the Chern character $\operatorname{ch}_{1}(E)$ in $N_{1}\left(X_{0} / Y_{0}\right)$. It is well known that the pairing

$$
N_{1}\left(X_{0} / Y_{0}\right) \times N_{1}\left(X_{0} / Y_{0}\right) \ni\left(D_{1}, D_{2}\right) \longmapsto D_{1} \cdot D_{2} \in \mathbb{R}
$$

is negative definite. Therefore there are only a finite number of possibilities for $r_{i} \in \mathbb{Z}_{\geq 0}$ which satisfy

$$
-\left(\sum_{i=1}^{N} r_{i} C_{i}\right)^{2} \leq 2
$$

and this shows the lemma.

Let us take $K>0$ as in Lemma 6.3. and define $\mathcal{S} \subset \mathrm{Coh}_{C}(X)$ to be

$$
\mathcal{S}:=\left\{E \in \operatorname{Coh}_{C}(X) \mid \operatorname{ch}_{2}(E)=\sum_{i=1}^{N} r_{i} C_{i}, \text { with } 0 \leq r_{i} \leq K\right\} .
$$

Proof of Lemma 4.5 .

For $n \in \mathbb{N}$, let $\omega_{n}$ be

$$
\omega_{n}:=\omega+\frac{1}{n} D_{i} \in A(X / Y)
$$

Because $\omega_{n}$ is ample, one has the stability condition

$$
\sigma_{n}:=\left(Z_{\left(\beta, \omega_{n}\right)}, \operatorname{Coh}_{C}(X)\right) \in U_{X},
$$

constructed in Lemma 4.1. It suffices to show $\sigma_{n}$ converges to $\sigma$.

For $E \in \mathcal{A}_{(i, k)}$, we denote by $\phi(E) \in(0,1]$ the phase with respect to the stability function $Z_{(\beta, \omega)}$ of Proposition 2.2. Also for a $\sigma$-semistable object $E \in \mathcal{D}_{X / Y}$, we use the same notation for its phase $\phi(E) \in \mathbb{R}$. Of course these two notions are compatible; i.e. if $E \in \mathcal{A}_{(i, k)}$ is $\sigma$-semistable, the above two phases $\phi(E)$ coincide. Similarly, we use the notation $\phi_{n}\left(E^{\prime}\right)$ for the phase of $E^{\prime} \in \operatorname{Coh}_{C}(X)$ with respect to $Z_{\left(\beta, \omega_{n}\right)}$ and the $\sigma_{n}$-semistable object $E^{\prime} \in \mathcal{D}_{X / Y}$.

Note that $\omega_{n}$ converges to $\omega$. Hence by the topology on $\operatorname{Stab}(X / Y)$ introduced in [5. Section 6], and using [5. Lemma 6.1], it suffices to show the following: for any $\varepsilon>0$, there exists $M>0$ such that $n>M$ implies:

for any $\sigma$-stable object $E \in \mathcal{A}_{(i, k)}$, if $F_{n}, F_{n}^{\prime} \in \mathcal{D}_{X / Y}$ are the semistable factors

of $E$ in $\sigma_{n}$ whose phases are the largest and the smallest, respectively, one has $\left|\phi_{n}\left(F_{n}\right)-\phi(E)\right|<\varepsilon, \quad\left|\phi_{n}\left(F_{n}^{\prime}\right)-\phi(E)\right|<\varepsilon$. 
For simplicity we treat the case of $k=0$. Other cases are similarly discussed. We divide the proof into two steps.

Step 1. There exists $M>0$ such that (3) holds for any $n>M$ and any $\sigma$-stable object $E \in \mathcal{A}_{(\beta, \omega)}$ with $\phi(E)=1$.

Proof. By Lemma 3.8 (i), any object $E \in \mathcal{A}_{(i, 0)}$ is given by a successive extension of the objects in

$$
\operatorname{Coh}_{\cup_{j \neq i} C_{j}}(X), \quad \mathcal{O}_{C_{i}}(-2)[1], \quad \mathcal{O}_{C_{i}}(-1) .
$$

Note that any sheaf $F \in \operatorname{Coh}_{\cup_{j \neq i} C_{j}} X$ with $\operatorname{dim} \operatorname{Supp}(F)=1$ satisfies $\operatorname{Im} Z_{(\beta, \omega)}(F)$ $>0$ because $\omega \cdot C_{j}>0$ for $j \neq i$. Therefore if $E \in \mathcal{A}_{(i, 0)}$ satisfies $\operatorname{Im} Z_{(\beta, \omega)}(E)=0$, $E$ is given by a successive extension of the following objects:

$$
\left\{\mathcal{O}_{x} \mid x \in \bigcup_{j \neq i} C_{j}\right\}, \quad \mathcal{O}_{C_{i}}(-2)[1], \quad \mathcal{O}_{C_{i}}(-1) .
$$

Therefore any $\sigma$-stable object $E$ of $\phi(E)=1$ is one of the above objects. Note that these objects are also stable in $\sigma_{n}$. One can easily calculate $\phi_{n}\left(\mathcal{O}_{x}\right)=\phi\left(\mathcal{O}_{x}\right)=1$ and

$\lim _{n \rightarrow \infty} \phi_{n}\left(\mathcal{O}_{C_{i}}(-2)[1]\right)=\phi\left(\mathcal{O}_{C_{i}}(-2)[1]\right)=1, \lim _{n \rightarrow \infty} \phi_{n}\left(\mathcal{O}_{C_{i}}(-1)\right)=\phi\left(\mathcal{O}_{C_{i}}(-1)\right)=1$.

Therefore it is enough to choose $M$ so that $n>M$ implies

$$
\left|\phi_{n}\left(\mathcal{O}_{C_{i}}(-2)[1]\right)-\phi\left(\mathcal{O}_{C_{i}}(-2)[1]\right)\right|<\varepsilon, \quad\left|\phi_{n}\left(\mathcal{O}_{C_{i}}(-1)\right)-\phi\left(\mathcal{O}_{C_{i}}(-1)\right)\right|<\varepsilon .
$$

Step 2. There exists $M^{\prime}>0$ such that (3) holds for any $n>M^{\prime}$ and any $\sigma$-stable object $E \in \mathcal{A}_{(i, 0)}$ with $0<\phi(E)<1$.

Proof. Let us take a $\sigma$-stable object $E \in \mathcal{A}_{(i, 0)}$ with $0<\phi(E)<1$. Then we have the exact sequence in $\mathcal{A}_{(i, 0)}$ by Lemma 6.2 (i),

$$
0 \longrightarrow H^{-1}(E)[1] \longrightarrow E \longrightarrow H^{0}(E) \longrightarrow 0 .
$$

By Lemma $6.2(\mathrm{i}), H^{-1}(E)$ is supported on $C_{i}$. Therefore $\phi\left(H^{-1}(E)[1]\right)=1$ and the stability of $E$ implies $H^{-1}(E)[1]=0$. Therefore we have

$$
E \in \mathcal{A}_{(i, 0)} \cap \operatorname{Coh}_{C}(X) .
$$

Let $F_{n} \in \operatorname{Coh}_{C}(X)$ be the semistable factor of $E$ in $\sigma_{n}$ of the largest phase. We have the exact sequence in $\operatorname{Coh}_{C}(X)$,

$$
0 \longrightarrow F_{n} \longrightarrow E \longrightarrow G_{n} \longrightarrow 0
$$

with

$$
\phi_{n}\left(F_{n}\right) \geq \phi_{n}(E) .
$$

By Lemma 6.2 (ii), we have the exact sequence in $\operatorname{Coh}_{C}(X)$,

$$
0 \longrightarrow H_{n} \longrightarrow F_{n} \longrightarrow I_{n} \longrightarrow 0
$$

such that

$$
H_{n} \in \mathcal{A}_{(i, 0)} \cap \operatorname{Coh}_{C}(X), \quad I_{n}[1] \in{ }^{0} \operatorname{Per}\left(\mathcal{D}_{X / Y_{i}}\right) .
$$

Here we assume $H_{n} \neq 0$ and $I_{n} \neq 0$. In the case that one of $H_{n}$ or $I_{n}$ is zero, one can argue similarly, and in fact it is easier. So we leave it to the reader. Combining the exact sequences (4) and (6), one gets the exact sequence

$$
0 \longrightarrow H_{n} \longrightarrow E \longrightarrow G_{n}^{\prime} \longrightarrow 0 \text {. }
$$


Note that $E \in \mathcal{A}_{(i, 0)} \subset{ }^{0} \operatorname{Per}\left(X / Y_{i}\right)$. Thus Lemma 3.5(ii) implies $R^{1} g_{i *} E=0$. Therefore the long exact sequence associated to (7) implies $R^{1} g_{i *} G_{n}^{\prime}=0$. Again by Lemma 3.5 we have $G_{n}^{\prime} \in{ }^{0} \operatorname{Per}\left(X / Y_{i}\right)$. This means the exact sequence (7) is also an exact sequence in $\mathcal{A}_{(i, 0)}$. By the stability of $E$, one gets

$$
\phi\left(H_{n}\right)<\phi(E) .
$$

Then we apply Lemma 6.4 below. Note that because $E$ is $\sigma$-stable, we have $\operatorname{Hom}(E, E)=\mathbb{C}$. Therefore Lemma 6.3 implies $E \in \mathcal{S}$, and because $H_{n}$ is a subsheaf of $E$, we have $H_{n} \in \mathcal{S}$. Furthermore, since $E$ and $H_{n}$ are objects of $\mathcal{A}_{(i, 0)}$, one has

$$
Z_{(\beta, \omega)}(E) \neq 0, \quad Z_{(\beta, \omega)}\left(H_{n}\right) \neq 0 .
$$

Let us take $\varepsilon^{\prime}=\frac{1}{3} \varepsilon$. Then by Lemma 6.4, we can find $M^{\prime}>0$ which does not depend on $E$, such that $n>M^{\prime}$ implies

$$
\left|\phi_{n}(E)-\phi(E)\right|<\varepsilon^{\prime}, \quad\left|\phi_{n}\left(H_{n}\right)-\phi\left(H_{n}\right)\right|<\varepsilon^{\prime} .
$$

Because $I_{n}[1] \in{ }^{0} \operatorname{Per}\left(\mathcal{D}_{X / Y_{i}}\right)$, we have

$$
Z_{(\beta, \omega)}\left(I_{n}[1]\right) \in \mathbb{R}<0 .
$$

Thus we apply Lemma 6.4 to get $0<\phi_{n}\left(I_{n}\right)<\varepsilon^{\prime}$ for $n>M^{\prime}$. Combined with the sequence (6), we have either

$$
0<\phi_{n}\left(F_{n}\right)<\varepsilon^{\prime}
$$

or

$$
\phi_{n}\left(H_{n}\right) \geq \phi_{n}\left(F_{n}\right)
$$

In the first case, we have

$$
\begin{aligned}
\left|\phi_{n}\left(F_{n}\right)-\phi(E)\right| & \leq\left(\phi_{n}\left(F_{n}\right)-\phi_{n}(E)\right)+\left|\phi_{n}(E)-\phi(E)\right| \\
& <2 \varepsilon^{\prime}<\varepsilon .
\end{aligned}
$$

Here we have used (5), (9) and (10). In the second case, we have

$$
\begin{aligned}
\left|\phi_{n}\left(F_{n}\right)-\phi(E)\right| & \leq\left(\phi_{n}\left(F_{n}\right)-\phi_{n}(E)\right)+\left|\phi_{n}(E)-\phi(E)\right| \\
& <\left(\phi_{n}\left(H_{n}\right)-\phi_{n}(E)\right)+\varepsilon^{\prime} \quad \text { from }(11),(9) \\
& <\left(\phi\left(H_{n}\right)-\phi(E)\right)+2 \varepsilon^{\prime}+\varepsilon^{\prime} \quad \text { from }(9) \\
& <3 \varepsilon^{\prime}=\varepsilon \quad \text { from }(8) .
\end{aligned}
$$

By the same argument, one can show $\left|\phi_{n}\left(F_{n}^{\prime}\right)-\phi(E)\right|<\varepsilon$ for the semistable factor $F_{n}^{\prime}$ in $\sigma^{\prime}$ with the smallest phase, when $n>M^{\prime}$. Therefore the condition (3) holds.

Here we have used the following lemma:

Lemma 6.4. We fix $\beta+i \omega \in \mathcal{W}_{i}$ as in Proposition 4.4. Then for each $\varepsilon^{\prime}>0$, there exists $M^{\prime}>0$ such that $n>M^{\prime}$ implies

$$
\sup \left\{\left|\frac{Z_{\left(\beta, \omega_{n}\right)}(F)}{Z_{(\beta, \omega)}(F)}-1\right|: F \in \mathcal{S}, Z_{(\beta, \omega)}(F) \neq 0\right\}<\varepsilon^{\prime} .
$$


Proof. One can calculate as follows:

$$
\left|\frac{Z_{\left(\beta, \omega_{n}\right)}(F)}{Z_{(\beta, \omega)}(F)}-1\right|=\frac{1}{n} \cdot \frac{\operatorname{ch}_{2} F \cdot D_{i}}{\left|-\operatorname{ch}_{3} F+\beta \operatorname{ch}_{2} F+i \omega \operatorname{ch}_{2} F\right|} .
$$

If $Z_{(\beta, \omega)}(F) \neq 0$, then $\omega \cdot \operatorname{ch}_{2}(F) \neq 0$ or $-\operatorname{ch}_{3} F+\beta \operatorname{ch}_{2} F \neq 0$. In the former case, one has

$$
\text { (12) } \leq \frac{1}{n} \cdot \frac{D_{i} \cdot \operatorname{ch}_{2} F}{\omega \cdot \operatorname{ch}_{2} F}
$$

and there are only a finite number of possibilities for the values

$$
\left\{\frac{D_{i} \cdot \operatorname{ch}_{2} F}{\omega \cdot \operatorname{ch}_{2} F}: F \in \mathcal{S}, \operatorname{ch}_{2} F \cdot \omega \neq 0\right\} .
$$

In the latter case, one has

$$
(12) \leq \frac{1}{n} \cdot \frac{D_{i} \cdot \operatorname{ch}_{2} F}{\left|-\operatorname{ch}_{3} F+\beta \operatorname{ch}_{2} F\right|},
$$

and because there are a finite number of possibilities for the values $\left\{\beta \cdot \operatorname{ch}_{2} F: F \in\right.$ $\mathcal{S}\}$, we have

$$
\inf \left\{\left|-\operatorname{ch}_{3} F+\beta \operatorname{ch}_{2} F\right|: F \in \mathcal{S},-\operatorname{ch}_{3} F+\beta \operatorname{ch}_{2} F \neq 0\right\}>0 .
$$

Therefore there exists a constant $K^{\prime}$ independent of $F \in \mathcal{S}$ with $Z_{(\beta, \omega)}(F) \neq 0$ such that $(12) \leq K^{\prime} / n$. Hence for each $\varepsilon^{\prime}$, one can take $M^{\prime}$ to be $K^{\prime} / \varepsilon$.

\section{ACKNOWLEDGEMENT}

This paper was written while the author was visiting the University of Sheffield from September 2005 to December 2005. The author thanks Tom Bridgeland for leading the author to the stability conditions and for many useful discussions and comments. In particular, the idea of constructing the stability conditions in Lemma 4.1 is due to him. The author also thanks his advisor professor Yujiro Kawamata, who recommended him to visit the University of Sheffield. Finally the author thanks the referee for reading the manuscript carefully and for giving him several nice comments to make the manuscript more readable. The author is supported by the Japan Society for the Promotion of Sciences Research Fellowships for Young Scientists, No. 1611452.

\section{REFERENCES}

[1] P. Aspinwall. A Point's Point of View of Stringy Geometry. preprint, pp. 1-16, 2002. hepth/0203111.

[2] A. Bergman. Stability conditions and Branes at Singularities. preprint. math.AG/0702092.

[3] A. Bondal and D. Orlov. Semiorthgonal decomposition for algebraic varieties. preprint, pp. 1-55, 1995. math.AG/9506012.

[4] T. Bridgeland. Spaces of stability conditions. preprint. math.AG/0611510.

[5] T. Bridgeland. Stability conditions on triangulated categories. to appear in Ann of Math. math.AG/0212237.

[6] T. Bridgeland. Flops and derived categories. Invent. Math, Vol. 147, pp. 613-632, 2002. MR.1893007 (2003h:14027)

[7] T. Bridgeland. Stability conditions on $K 3$ surfaces. preprint, pp. 1-41, 2003. math.AG/ 0307164 .

[8] T. Bridgeland. Stability conditions and Kleinian singularities. preprint, pp. 1-13, 2005. math.AG/0508257.

[9] T. Bridgeland. Derived categories of coherent sheaves. Proceedings of the 2006 ICM, Vol. II, pp. 563-582, 2006. MR2275610 (2007m:14018) 
[10] T. Bridgeland. Stability conditions on a non-compact Calabi-Yau threefold. Comm. Math. Phys, Vol. 266, pp. 715-733, 2006. MR2238896 (2007d:14075)

[11] J-C. Chen. Flops and equivalences of derived categories for three-folds with only Gorenstein singularities. J. Differential Geom., Vol. 61, pp. 227-261, 2002. MR1972146 (2004d:14012)

[12] M. Van den Bergh. Three-dimensional flops and noncommutative rings. Duke Math. J., Vol. 122, pp. 423-455, 2004. MR2057015 (2005e:14023)

[13] M. Douglas. D-branes, categories and $N=1$ supersymmetry. J. Math. Phys., Vol. 42, pp. 2818-2843, 2001. MR1840318 (2003b:81158)

[14] M. Douglas. Dirichlet branes, homological mirror symmetry, and stability. Proceedings of the 1998 ICM, pp. 395-408, 2002. MR1957548(2004c:81200)

[15] W. Fulton. Intersection theory, second edition, Vol. 2 of Ergebnisse der Mathematik und ihrer Grenzgebiete,3.Folge. Springer-Verlag, Berlin, 1998. MR.1644323 (99d:14003)

[16] D. Happel, I. Reiten, and S.O. Smalø. Tilting in abelian categories and quasitilted algebras. Mem. Amer. Math. Soc., Vol. 120, 1996. MR1327209 (97j:16009)

[17] A. Ishii and H. Uehara. Autoequivalences of derived categories on the minimal resolutions of $A_{n}$-singularities on surfaces. J. Differential Geom., Vol. 71, pp. 385-435, 2005. MR2198807 (2006k:14024)

[18] A. Ishii, K. Ueda, and H. Uehara. Stability Conditions on $A_{n}$-Singularities. pp. 1-44, 2006.

[19] Y. Kawamata. On the cone of divisors of Calabi-Yau fiber spaces. Internat. J. Math., Vol. 5, pp. 665-687, 1997. MR.1468356 (98g:14043)

[20] Y. Kawamata. D-equivalence and K-equivalence. J. Differential Geom., Vol. 61, pp. 147-171, 2002. MR:1949787 (2004m:14025)

[21] Y. Kawamata. Log crepant birational maps and derived categories. J. Math. Sci. Univ. Tokyo, Vol. 12, pp. 1-53, 2005. MR2150737 (2006a:14021)

[22] Y. Kawamata, K. Matsuda, and K. Matsuki. Introduction to the Minimal Model Problem. Adv. Stud. Pure Math., Vol. 10, pp. 283-360, 1987. MR946243 (89e:14015)

[23] Y. Kawamata and K. Matsuki. The number of minimal models for a 3-fold of general type is finite. Math. Ann., Vol. 276, pp. 595-598, 1987. MR879538 (88f:14012)

[24] J. Kollár. Flops. Nagoya Math. J., Vol. 113, pp. 15-36, 1989. MR986434 (90e:14011)

[25] J. Kollár and S. Mori. Birational geometry of algebraic varieties, Vol. 134 of Cambridge Tracts in Mathematics. Cambridge University Press, 1998. MR.1658959 (2000b:14018)

[26] E. Macri. Some examples of moduli spaces of stability conditions on derived categories. preprint. math.AG/0411613.

[27] S. Okada. Stability manifold of $\mathbb{P}^{1}$. J. Algebraic Geom., Vol. 15, pp. 487-505, 2006. MR2219846 (2007b:14036)

[28] M. Reid. Minimal models of canonical 3-folds. Algebraic Varieties and Analytic Varieties (S. Iitaka, ed.), Adv. Stud. Pure Math., Kinokuniya, Tokyo, and North-Holland, Amsterdam, Vol. 1, pp. 131-180. MR.715649 (86a:14010)

[29] R. Thomas. Stability conditions and the braid groups. Comm. Anal. Geom., Vol. 14, pp. 135-161, 2006. MR2230573 (2007j:53113)

Graduate School of Mathematical Sciences, University of Tokyo, 3-8-1 Komaba, MEguro, TOKYO, JAPAN

E-mail address: toda@ms.u-tokyo.ac.jp

Current address: Institute for the Physics and Mathematics of the Universe, University of Tokyo, 5-1-5 Kashiwanoha, Kashiwa City, Chiba, Japan 